\title{
Small Perturbations for Nonlinear Schrödinger Equations with Magnetic Potential
}

\author{
Youpei Zhang, Xianhua Tang and Vicenţiu D. Rădulescu*
}

\begin{abstract}
We are concerned with the qualitative analysis of solutions for three classes of nonlinear problems driven by the magnetic Laplace operator. We are mainly interested in the perturbation effects created by two reaction terms with different structure. Two equations are studied on bounded domains (under Dirichlet boundary condition) while the third problem is on the entire Euclidean space. Our main results establish that if a certain perturbation is sufficiently small (in a prescribed sense) then the problems have at least two distinct solutions in a related magnetic Sobolev space. The proofs combine variational, topological and analytic methods.
\end{abstract}

Mathematics Subject Classification (2010). Primary 35Q55; Secondary 35J60, $58 \mathrm{E} 05$.

Keywords. Magnetic Laplace operator, critical Sobolev exponent, lack of compactness, magnetic Sobolev space, perturbation, constrained minimization, multiple solutions.

\section{Introduction}

In quantum mechanics, the Hamiltonian for a nonrelativistic charged particle in an electromagnetic field is defined by $(-i \nabla-A)^{2}+b$, where $b: D \subset \mathbb{R}^{N} \rightarrow \mathbb{R}$ is the electric (or scalar) potential, $A: D \subset \mathbb{R}^{N} \rightarrow \mathbb{R}^{N}$ and the open set $D \subset \mathbb{R}^{N}$ is the region that constrains the particles. The vector (magnetic) potential $A=$ $\left(A_{1}, A_{2}, \ldots, A_{N}\right)$ is a source for the magnetic field $B=\operatorname{curl} A$, where $\operatorname{curl} A$ is the $N \times N$ skew-symmetric matrix with entries $B_{j k}=\partial_{j} A_{k}-\partial_{k} A_{j}$. The magnetic potential $A$ can also be expressed in the language of differential forms, namely if $A$

* Corresponding author.

This work is partially supported by the National Science Foundation of China (No. 11971485), the China Scholarship Council (No. 201906370079) and the Fundamental Research Funds for the Central Universities of Central South University (No. 2019zzts211). 
is the 1 -form $A_{1} d x_{1}+\cdots+A_{N} d x_{N}$, then $B=d A$. Of course, if $N=3$, then $\operatorname{curl} A$ has also the usual representation as a vector in $\mathbb{R}^{3}$.

In this paper, we start by considering the subcritical perturbed problem

$$
\begin{cases}(-i \nabla-A(x))^{2} u=|u|^{q-2} u+\lambda f & \text { in } \Omega, \\ u=0 & \text { on } \partial \Omega,\end{cases}
$$

on a smooth bounded domain $\Omega$ in $\mathbb{R}^{N}$, where $\lambda$ is a positive parameter, $N \geq 2$, $2<q<2^{*}$, and $A: \Omega \subset \mathbb{R}^{N} \rightarrow \mathbb{R}^{N}$ is the magnetic potential. Here, $2^{*}$ denotes the critical Sobolev exponent, that is, $2^{*}=\frac{2 N}{N-2}$ if $N \geq 3$, and $2^{*}=+\infty$ if $N=2$.

Next, we are concerned with the sublinear perturbation of a critical problem

$$
\begin{cases}(-i \nabla-A(x))^{2} u=|u|^{2^{*}-2} u+\lambda|u|^{q-2} u & \text { in } \Omega, \\ u=0 & \text { on } \partial \Omega,\end{cases}
$$

where $\lambda$ is a positive parameter, $N \geq 3$ and $1<q<2$.

Finally, we study a perturbation problem in $\mathbb{R}^{N}$, but from another point of view. More exactly, we will analyze the effect of a small perturbation $g$ in the semilinear elliptic problem

$$
(-i \nabla-A(x))^{2} u+b(x) u=K(x)|u|^{q-2} u+g(x) \text { in } \mathbb{R}^{N},
$$

where $N \geq 2,2<q<2^{*}$, and $A: \mathbb{R}^{N} \rightarrow \mathbb{R}^{N}$ is the magnetic potential.

We assume that $A$ and $b, K \in L^{\infty}\left(\mathbb{R}^{N}, \mathbb{R}\right)$ satisfy the following conditions:

(A) For all $y \in \mathbb{Z}^{N}$ there exists $\varphi_{y} \in H_{\text {loc }}^{1}\left(\mathbb{R}^{N}, \mathbb{R}\right)$ such that $A(x+y)-A(x)=$ $\nabla \varphi_{y}(x)$

(B) ess $\lim _{|x| \rightarrow+\infty} b(x)=b_{\infty} \in \mathbb{R}_{+}$and there exists a constant $b_{1}>0$ such that $b_{1} \leq b(x) \leq b_{\infty}$ a.e. in $\mathbb{R}^{N}$;

$(K)$ ess $\lim _{|x| \rightarrow+\infty} K(x)=K_{\infty} \in \mathbb{R}_{+}$and $K(x) \geq K_{\infty}$ a.e. in $\mathbb{R}^{N}$;

$(M) \operatorname{meas}\left(\left\{x \in \mathbb{R}^{N}: b(x)<b_{\infty}\right\} \bigcup\left\{x \in \mathbb{R}^{N}: K(x)>K_{\infty}\right\}\right)>0$.

We recall that the Schrödinger operator with magnetic potential is defined by

$$
(-i \nabla-A(z))^{2} \xi=-\Delta \xi+2 i A \nabla \xi+|A|^{2} \xi+i \xi \operatorname{div} A .
$$

The problems we study in this paper are related with the existence of solitary waves, namely solutions of the form $\phi(z, t):=e^{-i \frac{E}{\hbar} t} u(z)$, with $E \in \mathbb{R}$, for the nonlinear Schrödinger equation

$$
i \hbar \frac{\partial \phi}{\partial t}=\left(\frac{\hbar}{i} \nabla-A(z)\right)^{2} \phi+U(z) \phi-l\left(|\phi|^{2}\right) \phi, \quad z \in D,
$$

where $t>0, N \geq 2, \hbar$ is the Planck constant, and $A$ is a magnetic potential associated with a given magnetic field $B, U(z)$ is a real electric potential and the nonlinear term $l$ is a superlinear function. A direct calculation states that $\phi$ is a solitary wave of problem (4) if and only if $u$ is a solution of the problem

$$
\left(\frac{\hbar}{i} \nabla-A(z)\right)^{2} u+b(z) u=l\left(|u|^{2}\right) u, \quad z \in D,
$$


where $b(z)=U(z)-E$. It is significant to study the existence and the shape of such solutions in the semiclassical limit, that is, as $\hbar \rightarrow 0$. The significance of this research relies on this fact that the transition from Quantum Mechanics to Classical Mechanics can be formally performed by sending the Planck constant to zero.

In the last decades, many authors have conducted extensive research on perturbations of problem (5) and of boundary value problems without magnetic potential (namely $A \equiv 0$ and $\hbar=1$ ). We first give the elementary example

$$
\begin{cases}-\Delta u=|u|^{q-2} u & \text { in } \Omega, \\ u=0 & \text { on } \partial \Omega,\end{cases}
$$

where $\Omega$ is a smooth bounded domain in $\mathbb{R}^{N}(N \geq 2)$ and $2<q<2^{*}$. A classical result, based on a $\mathbb{Z}_{2}$ symmetric version of the Mountain Pass Theorem (see Ambrosetti and Rabinowitz [3]), shows that problem (6) admits infinitely many solutions in $H_{0}^{1}(\Omega, \mathbb{R})$. A natural question is to see what happens if the above problem is affected by a certain perturbation. Consider the problem

$$
\begin{cases}-\Delta u=|u|^{q-2} u+\eta(x) & \text { in } \Omega, \\ u=0 & \text { on } \partial \Omega .\end{cases}
$$

Bahri-Berestycki [6] and Struwe [23] have proved independently that there exists $q_{0}<2^{*}$ such that for every $\eta \in L^{2}(\Omega)$, problem (7) still possesses infinitely many solutions, provided $2<q<q_{0}$. Furthermore, Bahri [5] has obtained that for any $2<q<2^{*}$ there is a dense open set of $\eta \in H^{-1}(\Omega, \mathbb{R})$ for which problem (7) admits infinitely many solutions.

To the best of our knowledge, the first result in the presence of a magnetic potential $A \not \equiv 0$ seems to be obtained by Esteban and Lions [12]. They have made use of the concentration-compactness principle and minimization arguments to obtain solution for $\hbar>0$ fixed and dimensions $N=2$ or $N=3$. We also would like to cite the papers $[1,11,4]$ for other results related to the problems (1) and (3) in the presence of magnetic field when the nonlinearity has a subcritical growth. We also refer to the papers $[2,4,13,14]$ for the critical case.

Now, we assume that $A, \widetilde{A} \in L_{\text {loc }}^{s}\left(\mathbb{R}^{N}, \mathbb{R}^{N}\right)$ for some $s \in[1, \infty)$ and $\operatorname{curl} A=$ $B=\operatorname{curl} \widetilde{A}$ (in the sense of distributions). Then $\widetilde{A}-A=\nabla \varphi$ for some $\varphi \in W_{\text {loc }}^{1, s}\left(\mathbb{R}^{N}\right)$, (see [17, Lemma 1.1]). We can easily deduce that if $\widetilde{u}=e^{i \varphi}$, then $\nabla_{\widetilde{A}} \widetilde{u}=e^{i \varphi} \nabla_{A} u$ and so $\int_{\mathbb{R}^{N}}\left|\nabla_{\widetilde{A}} \widetilde{u}\right|^{2} d x=\int_{\mathbb{R}^{N}}\left|\nabla_{A} u\right|^{2} d x$, where $\nabla_{A}=(-i \nabla-A)$ is given in Section 2 . The above properties are called the gauge invariance and the transformation $T: u \rightarrow \widetilde{u}$ the change of gauge. The condition $(A)$ is inspired by hypothesis A2 introduced by Arioli and Szulkin [4]. By hypothesis $(A)$, we can define a different "transformation" $T$ (see Subsection 5.1) satisfying the above gauge invariance.

Motivated by the works of Rădulescu-Smets [22] and Cîrstea-Rădulescu [10], we are concerned with the qualitative analysis of solutions for three classes of nonlinear problems driven by the magnetic Laplace operator. Due to the existence of the magnetic potential $A$, problems (1), (2) and (3) cannot be converted into pure real-valued problems, thus we are supposed to directly treat these problems with 
complex values. But in this way, we will encounter some new challenges when dealing with our problems. In particular, we refer to the combined effects of the lack of compactness of problems (2) and (3) and the presence of a magnetic potential. Since we deal with different problems where the functions are complex-valued, it is necessary to make a careful analysis of technical estimates used in this paper. By establishing new threshold estimates we overcome the lack of compactness in problem (2). Furthermore, we overcome the lack of compactness of problems (3) by employing a variant of the Mountain Pass theorem without the Palais-Smale condition (see Brezis and Nirenberg [9, Theorem 2.2]), combined with a generalization of the Brezis-Lieb lemma [8, Theorem 1]. We also refer to Pucci and Rădulescu [20] for a survey concerning the Mountain Pass theorem.

If we perturb problems (1), (2) and (3) such that the perturbations do not exceed some levels, then we will show in this paper that problems (1), (3) have at least two solutions while problem (2) has at least one nontrivial solution. More precisely, if $\lambda$ and $\|g\|_{b,-1}$ (see Section 2) are sufficiently small, then, problem (2) has a mountain pass solution, while problems (1), (3) have local minimums near the origin, whereas, the second solution is obtained as a mountain pass. Since problem (3) is investigated in $\mathbb{R}^{N}$, we need to take advantage of the hypotheses $(B),(K)$ and $(M)$ in order to conclude the existence of the mountain pass solution for problem (3), while the existence of a simple solution (the local minimum) will follow without these stronger assumptions for problem (3).

We refer to Laptev et al. $[15,16]$ for recent advances in the study of magnetic differential operators and to Papageorgiou, Rădulescu and Repovš [19] for some of the abstract methods used in this paper.

\section{Abstract framework and the main results}

In this section, we outline the variational framework and give the statement of the main results. We refer to Esteban-Lions in [12] and Lieb-Loss in [18] for more details on the complex-valued function Sobolev spaces with magnetic potential.

Let $U$ be an open set in $\mathbb{R}^{N}$. For $u: U \rightarrow \mathbb{C}$, we define

$$
\nabla_{A} u:=(-i \nabla-A) u \text {. }
$$

Let $\Omega$ be a domain with smooth boundary in $\mathbb{R}^{N}$, we shall denote by $H_{A}(\Omega)$ the Hilbert space obtained by the closure of $C_{0}^{\infty}(\Omega, \mathbb{C})$ under the scalar product

$$
\langle u, v\rangle_{\Omega}:=\operatorname{Re} \int_{\Omega} \nabla_{A} u \overline{\nabla v} d x,
$$

where $\operatorname{Re}(\omega)$ denotes the real part of $\omega \in \mathbb{C}, \bar{\omega}$ is its complex conjugated.

Moreover, we shall denote by $\|u\|_{A}$ the norm induced by this scalar product, that is,

$$
\|u\|_{A}:=\left(\int_{\Omega}\left|\nabla_{A} u\right|^{2} d x\right)^{\frac{1}{2}} .
$$


For $u: \mathbb{R}^{N} \rightarrow \mathbb{C}$, let us define

$$
H_{A}\left(\mathbb{R}^{N}\right):=\left\{u \in L^{2}\left(\mathbb{R}^{N}, \mathbb{C}\right):\left|\nabla_{A} u\right| \in L^{2}\left(\mathbb{R}^{N}, \mathbb{R}\right)\right\}
$$

and, for $N \geq 3$,

$$
\mathcal{D}_{A}^{1,2}\left(\mathbb{R}^{N}\right):=\left\{u \in L^{2^{*}}\left(\mathbb{R}^{N}, \mathbb{C}\right):\left|\nabla_{A} u\right| \in L^{2}\left(\mathbb{R}^{N}, \mathbb{R}\right)\right\} .
$$

The spaces $H_{A}\left(\mathbb{R}^{N}\right)$ and $\mathcal{D}_{A}^{1,2}\left(\mathbb{R}^{N}\right)$ are Hilbert spaces endowed with scalar product, respectively,

$$
\langle u, v\rangle_{\mathbb{R}^{N}}:=\operatorname{Re} \int_{\mathbb{R}^{N}}\left(\nabla_{A} u \overline{\nabla_{A} v}+u \bar{v}\right) d x
$$

and

$$
\langle u, v\rangle_{\mathcal{D}}:=\operatorname{Re} \int_{\mathbb{R}^{N}} \nabla_{A} u \overline{\nabla_{A} v} d x .
$$

Let $\|u\|_{H_{A}\left(\mathbb{R}^{N}\right)}$ and $\|u\|_{\mathcal{D}_{A}^{1,2}\left(\mathbb{R}^{N}\right)}$ denote the norms induced by the scalar products, namely

and

$$
\|u\|_{H_{A}\left(\mathbb{R}^{N}\right)}:=\left(\int_{\mathbb{R}^{N}}\left(\left|\nabla_{A} u\right|^{2}+|u|^{2}\right) d x\right)^{\frac{1}{2}}
$$

$$
\|u\|_{\mathcal{D}_{A}^{1,2}\left(\mathbb{R}^{N}\right)}:=\left(\int_{\mathbb{R}^{N}}\left|\nabla_{A} u\right|^{2} d x\right)^{\frac{1}{2}} .
$$

By [12, Section 2] and [18, Theorem 7.22], $C_{0}^{\infty}\left(\mathbb{R}^{N}, \mathbb{C}\right)$ is dense in $H_{A}\left(\mathbb{R}^{N}\right)$ and $\mathcal{D}_{A}^{1,2}\left(\mathbb{R}^{N}\right)$ (in [12], the function space $\mathcal{D}_{A}^{1,2}\left(\mathbb{R}^{N}\right)$ has been defined as the closure of $C_{0}^{\infty}\left(\mathbb{R}^{N}, \mathbb{C}\right)$ with respect to the norm $\left.\|u\|_{\mathcal{D}_{A}^{1,2}\left(\mathbb{R}^{N}\right)}\right)$. Finally, the spaces $H_{A}\left(\mathbb{R}^{N}\right)$ and $H^{1}\left(\mathbb{R}^{N}, \mathbb{C}\right)$ are incomparable, more precisely, in general $H_{A}\left(\mathbb{R}^{N}\right) \nsubseteq H^{1}\left(\mathbb{R}^{N}, \mathbb{C}\right)$ and $H^{1}\left(\mathbb{R}^{N}, \mathbb{C}\right) \nsubseteq H_{A}\left(\mathbb{R}^{N}\right)$.

Let $H_{b}\left(\mathbb{R}^{N}\right)$ and $H_{b_{\infty}}\left(\mathbb{R}^{N}\right)$ be the Sobolev spaces defined as the completion of $C_{0}^{\infty}\left(\mathbb{R}^{N}, \mathbb{C}\right)$ with respect to the norms

$$
\|u\|_{b}:=\left(\int_{\mathbb{R}^{N}}\left(\left|\nabla_{A} u\right|^{2}+b(x)|u|^{2}\right) d x\right)^{\frac{1}{2}}
$$

and

$$
\|u\|_{b_{\infty}}:=\left(\int_{\mathbb{R}^{N}}\left(\left|\nabla_{A} u\right|^{2}+b_{\infty}|u|^{2}\right) d x\right)^{\frac{1}{2}},
$$

respectively. The spaces $H_{b}\left(\mathbb{R}^{N}\right)$ and $H_{b_{\infty}}\left(\mathbb{R}^{N}\right)$ are Hilbert spaces endowed with scalar products

$$
\langle u, v\rangle_{b}:=\operatorname{Re} \int_{\mathbb{R}^{N}}\left(\nabla_{A} u \overline{\nabla_{A} v}+b(x) u \bar{v}\right) d x
$$

and

$$
\langle u, v\rangle_{b_{\infty}}:=\operatorname{Re} \int_{\mathbb{R}^{N}}\left(\nabla_{A} u \overline{\nabla_{A} v}+b_{\infty} u \bar{v}\right) d x
$$

respectively. 
We shall denote by $\|\cdot\|_{-1}$ and $\|\cdot\|_{b,-1}$ the norms of $H_{A}^{-1}(\Omega)$ and $H_{b}^{-1}\left(\mathbb{R}^{N}\right)$, respectively, where

$$
H_{A}^{-1}(\Omega)=\left(H_{A}(\Omega)\right)^{*} \text { and } H_{b}^{-1}\left(\mathbb{R}^{N}\right)=\left(H_{b}\left(\mathbb{R}^{N}\right)\right)^{*} .
$$

Throughout this work we suppose that $f \in H_{A}^{-1}(\Omega) \backslash\{0\}$ and $g \in H_{b}^{-1}\left(\mathbb{R}^{N}\right) \backslash\{0\}$.

Definition 2.1. We say that a function $u \in H_{A}(\Omega)$ is a weak solution of (1) if

$$
\operatorname{Re}\left(\int_{\Omega} \nabla_{A} u \overline{\nabla_{A} v} d x-\int_{\Omega}|u|^{q-2} u \bar{v} d x-\int_{\Omega} \lambda f \bar{v} d x\right)=0,
$$

for all $v \in H_{A}(\Omega)$.

Definition 2.2. We say that a function $u \in H_{A}(\Omega)$ is a weak solution of (2) if

$$
\operatorname{Re}\left(\int_{\Omega} \nabla_{A} u \overline{\nabla_{A} v} d x-\int_{\Omega}|u|^{2^{*}-2} u \bar{v} d x-\lambda \int_{\Omega}|u|^{q-2} u \bar{v} d x\right)=0,
$$

for all $v \in H_{A}(\Omega)$.

Definition 2.3. We say that $u \in H_{b}\left(\mathbb{R}^{N}\right)$ is a weak solution of (3) if

$$
\operatorname{Re}\left(\int_{\mathbb{R}^{N}}\left(\nabla_{A} u \overline{\nabla_{A} v}+b(x) u \bar{v}\right) d x-\int_{\mathbb{R}^{N}} K(x)|u|^{q-2} u \bar{v} d x-\int_{\mathbb{R}^{N}} g \bar{v} d x\right)=0,
$$

for all $v \in H_{b}\left(\mathbb{R}^{N}\right)$.

Our main results are the following.

Theorem 2.4. Let $2<q<2^{*}$. Then, for each $f \in H_{A}^{-1}(\Omega) \backslash\{0\}$, there exists $C_{1}>0$ such that for all $0<\lambda<\frac{C_{1}}{\|f\|_{-1}}$, problem (1) has at least two solutions.

Theorem 2.5. There exists $\lambda^{*}>0$ such that problem (2) has at least one nontrivial solution for all $\lambda \in\left(0, \lambda^{*}\right)$.

Theorem 2.6. Let $2<q<2^{*}$ and conditions $(A),(B),(K)$ and $(M)$ are fulfilled. Then there exists $C^{\prime}>0$ such that problem (3) has at least two solutions, for any $g \in H_{b}^{-1}\left(\mathbb{R}^{N}\right) \backslash\{0\}$ satisfying $\|g\|_{b,-1}<C^{\prime}$.

\subsection{Auxiliary properties}

Weak solutions of problems (1), (2) and (3) correspond to the critical points of the following energy functionals

$$
\begin{array}{ll}
J(u)=\frac{1}{2} \int_{\Omega}\left|\nabla_{A} u\right|^{2} d x-\frac{1}{q} \int_{\Omega}|u|^{q} d x-\operatorname{Re} \int_{\Omega} \lambda f \bar{u} d x \quad \text { for all } u \in H_{A}(\Omega), \\
\mathcal{J}(u)=\frac{1}{2} \int_{\Omega}\left|\nabla_{A} u\right|^{2} d x-\frac{1}{2^{*}} \int_{\Omega}|u|^{2^{*}} d x-\frac{\lambda}{q} \int_{\Omega}|u|^{q} d x \quad \text { for all } u \in H_{A}(\Omega),
\end{array}
$$

and

$$
\begin{aligned}
\Psi(u)= & \frac{1}{2} \int_{\mathbb{R}^{N}}\left(\left|\nabla_{A} u\right|^{2}+b(x)|u|^{2}\right) d x-\frac{1}{q} \int_{\mathbb{R}^{N}} K(x)|u|^{q} d x \\
& -\operatorname{Re} \int_{\mathbb{R}^{N}} g(x) \bar{u} d x \quad \text { for all } u \in H_{b}\left(\mathbb{R}^{N}\right),
\end{aligned}
$$


respectively.

The following well-known diamagnetic inequality is proved by Esteban-Lions in [12] and Lieb-Loss in [18].

Lemma 2.7. If $u \in H_{A}(\Omega)$ (resp. $\left.u \in H_{A}\left(\mathbb{R}^{N}\right)\right)$, then $|u| \in H_{0}^{1}(\Omega, \mathbb{R})$ (resp. $|u| \in$ $\left.H^{1}\left(\mathbb{R}^{N}, \mathbb{R}\right)\right)$ and

$$
|\nabla| u|(x)|=\left|\operatorname{Re}\left(\nabla u \frac{\bar{u}}{|u|}\right)\right|=\left|\operatorname{Re}\left((\nabla u-i A u) \frac{\bar{u}}{|u|}\right)\right| \leq\left|\nabla_{A} u(x)\right|,
$$

for a.e. $x \in \Omega\left(\right.$ resp. $\left.x \in \mathbb{R}^{N}\right)$, where $|u|(\cdot):=|u(\cdot)|$.

Furthermore, the embedding $H_{A}(\Omega) \hookrightarrow L^{\mu}(\Omega, \mathbb{C})\left(\operatorname{resp} ., H_{A}\left(\mathbb{R}^{N}\right) \hookrightarrow L^{\mu}\left(\mathbb{R}^{N}, \mathbb{C}\right)\right)$ is continuous for each $1 \leq \mu \leq 2^{*}$ (for any $\mu \in[1,+\infty)$ if $N=2$ ) and it is compact (resp. local compact) for $1 \leq \mu<2^{*}$.

A direct calculation implies that $J, \mathcal{J} \in C^{1}\left(H_{A}(\Omega), \mathbb{R}\right)$ with

$$
\left\langle J^{\prime}(u), v\right\rangle=\operatorname{Re}\left(\int_{\Omega} \nabla_{A} u \overline{\nabla_{A} v} d x-\int_{\Omega}|u|^{q-2} u \bar{v} d x-\int_{\Omega} \lambda f \bar{v} d x\right)
$$

and

$$
\left\langle\mathcal{J}^{\prime}(u), v\right\rangle=\operatorname{Re}\left(\int_{\Omega} \nabla_{A} u \overline{\nabla_{A} v} d x-\int_{\Omega}|u|^{2^{*}-2} u \bar{v} d x-\lambda \int_{\Omega}|u|^{q-2} u \bar{v} d x\right)
$$

for all $v \in H_{A}(\Omega)$. Thus, the weak solutions of problems (1) and (2) are precisely the critical points of $J$ and $\mathcal{J}$, respectively.

The following result shows that $H_{b}\left(\mathbb{R}^{N}\right)$ is continuously embedded in $L^{q}\left(\mathbb{R}^{N}, \mathbb{C}\right)$. Applying this fact and $(K)$ we deduce that the functional $\Psi$ is welldefined in $H_{b}\left(\mathbb{R}^{N}\right)$.

Proposition 2.8. There exists a positive $M_{q}>0$ such that, for any $u \in H_{b}\left(\mathbb{R}^{N}\right)$,

$$
\left(\int_{\mathbb{R}^{N}}|u|^{q} d x\right)^{\frac{1}{q}} \leq M_{q}\left(\int_{\mathbb{R}^{N}}\left(\left|\nabla_{A} u\right|^{2}+b(x)|u|^{2} d x\right)^{\frac{1}{2}} .\right.
$$

Proof. By Lemma 2.7 and the hypotheses of $b$, it is obvious that Proposition 2.8 holds true. This proof is now complete.

In this paper we denote by " - " the weak convergence and by " $\rightarrow$ " the strong convergence in an arbitrary Banach space $X$.

Remark 2.9. Let $\left\{u_{n}\right\}$ be a sequence that converges weakly to some $u_{0}$ in $H_{b}\left(\mathbb{R}^{N}\right)$. Since $b_{\infty} \geq b(x) \geq b_{1}>0$ a.e. in $\mathbb{R}^{N}$, we can easily see that the norms of the spaces $H_{A}\left(\mathbb{R}^{N}\right), H_{b}\left(\mathbb{R}^{N}\right)$ and $H_{b_{\infty}}\left(\mathbb{R}^{N}\right)$ are equivalent. Hence, the embedding $H_{b}\left(\mathbb{R}^{N}\right) \hookrightarrow$ $L^{\mu}\left(\mathbb{R}^{N}, \mathbb{C}\right)$ is continuous for each $1 \leq \mu \leq 2^{*}$ (for any $\mu \in[1,+\infty$ ) if $N=2$ ) and it is local compact for $1 \leq \mu<2^{*}$. Therefore, we may assume (up to a subsequence) that

$$
\begin{cases}u_{n} \rightarrow u_{0} & \text { in } H_{b}\left(\mathbb{R}^{N}\right), \\ u_{n} \rightarrow u_{0} & \text { in } L_{\mathrm{loc}}^{\mu}\left(\mathbb{R}^{N}, \mathbb{C}\right) \\ u_{n} \rightarrow u_{0} & \text { a.e. in } \mathbb{R}^{N} .\end{cases}
$$


For all $u \in H_{b}\left(\mathbb{R}^{N}\right)$, we define the functionals $\Upsilon: H_{b}\left(\mathbb{R}^{N}\right) \rightarrow \mathbb{R}$ and $\Upsilon_{\infty}$ : $H_{b}\left(\mathbb{R}^{N}\right) \rightarrow \mathbb{R}$ by

$$
\Upsilon(u)=\frac{1}{2} \int_{\mathbb{R}^{N}}\left(\left|\nabla_{A} u\right|^{2}+b(x)|u|^{2}\right) d x-\frac{1}{q} \int_{\mathbb{R}^{N}} K(x)|u|^{q} d x
$$

and

$$
\Upsilon_{\infty}(u)=\frac{1}{2} \int_{\mathbb{R}^{N}}\left(\left|\nabla_{A} u\right|^{2}+b_{\infty}|u|^{2}\right) d x-\frac{1}{q} \int_{\mathbb{R}^{N}} K_{\infty}|u|^{q} d x,
$$

respectively. A direct calculation shows that $\Psi, \Upsilon, \Upsilon_{\infty} \in C^{1}\left(H_{b}\left(\mathbb{R}^{N}\right), \mathbb{R}\right)$ and their derivatives are given by

$$
\begin{aligned}
\left\langle\Psi^{\prime}(u), v\right\rangle= & \operatorname{Re}\left(\int_{\mathbb{R}^{N}}\left(\nabla_{A} u \overline{\nabla_{A v}}+b(x) u \bar{v}\right) d x-\int_{\mathbb{R}^{N}} K(x)|u|^{q-2} u \bar{v} d x\right. \\
& \left.-\int_{\mathbb{R}^{N}} g(x) \bar{v} d x\right) \\
\left\langle\Upsilon^{\prime}(u), v\right\rangle= & \operatorname{Re}\left(\int_{\mathbb{R}^{N}}\left(\nabla_{A} u \overline{\nabla_{A v}}+b(x) u \bar{v}\right) d x-\int_{\mathbb{R}^{N}} K(x)|u|^{q-2} u \bar{v} d x\right), \\
\left\langle\Upsilon_{\infty}^{\prime}(u), v\right\rangle= & \operatorname{Re}\left(\int_{\mathbb{R}^{N}}\left(\nabla_{A} u \overline{\nabla_{A} v}+b_{\infty} u \bar{v}\right) d x-\int_{\mathbb{R}^{N}} K_{\infty}|u|^{q-2} u \bar{v} d x\right),
\end{aligned}
$$

for all $u, v \in H_{b}\left(\mathbb{R}^{N}\right)$.

Brezis and Lieb established in [8, Theorem 1] a subtle refinement of Fatou's lemma. Our following result is a weighted variant of the Brezis-Lieb lemma. This proof uses some ideas found in Cîrstea and Rădulescu [10, Lemma 2]. We give the details of the proof for the convenience of the reader.

Lemma 2.10. Let $\left\{u_{n}\right\}$ be a sequence which is weakly convergent to $u_{0}$ in $H_{b}\left(\mathbb{R}^{N}\right)$. Then

$$
\lim _{n \rightarrow \infty} \int_{\mathbb{R}^{N}} K(x)\left(\left|u_{n}\right|^{q}-\left|u_{n}-u_{0}\right|^{q}\right) d x=\int_{\mathbb{R}^{N}} K(x)\left|u_{0}\right|^{q} d x .
$$

Proof. By Proposition 2.8 and the boundedness of $\left\{u_{n}\right\}$ in $H_{b}\left(\mathbb{R}^{N}\right)$ we can see that $\left\{u_{n}\right\}$ is a bounded sequence in $L^{q}\left(\mathbb{R}^{N}, \mathbb{C}\right)$. For given $\varepsilon>0$ we take $R^{\varepsilon}>0$ such that

$$
\int_{|x|>R^{\varepsilon}} K(x)\left|u_{0}\right|^{q} d x<\varepsilon
$$

We first observe that

$$
\begin{aligned}
& \left|\int_{\mathbb{R}^{N}} K(x)\left(\left|u_{n}\right|^{q}-\left|u_{0}\right|^{q}-\left|u_{n}-u_{0}\right|^{q}\right) d x\right| \\
= & \mid \int_{|x| \leq R^{\varepsilon}} K(x)\left(\left|u_{n}\right|^{q}-\left|u_{0}\right|^{q}\right) d x-\int_{|x| \leq R^{\varepsilon}} K(x)\left(\left|u_{n}-u_{0}\right|^{q}\right) d x \\
& -\int_{|x|>R^{\varepsilon}} K(x)\left|u_{0}\right|^{q}+\int_{|x|>R^{\varepsilon}} K(x)\left(\left|u_{n}\right|^{q}-\left|u_{n}-u_{0}\right|^{q}\right) d x \mid
\end{aligned}
$$




$$
\begin{aligned}
\leq \mid & \int_{|x| \leq R^{\varepsilon}} K(x)\left(\left|u_{n}\right|^{q}-\left|u_{0}\right|^{q}\right) d x \mid+\int_{|x| \leq R^{\varepsilon}} K(x)\left(\left|u_{n}-u_{0}\right|^{q}\right) d x \\
& +\int_{|x|>R^{\varepsilon}} K(x)\left|u_{0}\right|^{q} d x+\int_{|x|>R^{\varepsilon}} q K(x)\left|\theta u_{0}+\left(u_{n}-u_{0}\right)\right|^{q-1}\left|u_{0}\right| d x,
\end{aligned}
$$

where $0 \leq \theta(x) \leq 1$. On the other hand, by relation (10) and Hölder's inequality we can deduce that

$$
\begin{aligned}
& \int_{|x|>R^{\varepsilon}} K(x)\left|\theta u_{0}+\left(u_{n}-u_{0}\right)\right|^{q-1}\left|u_{0}\right| d x \\
\leq & c \int_{|x|>R^{\varepsilon}} K(x)\left(\left|u_{0}\right|^{q}+\left|u_{n}-u_{0}\right|^{q-1}\left|u_{0}\right|\right) d x \\
\leq & c\left[\left(\int_{|x|>R^{\varepsilon}} K(x)\left|u_{n}-u_{0}\right|^{q} d x\right)^{(q-1) / q}\right. \\
& \left.\times\left(\int_{|x|>R^{\varepsilon}} K(x)\left|u_{0}\right|^{q} d x\right)^{1 / q}+\int_{|x|>R^{\varepsilon}} K(x)\left|u_{0}\right|^{q} d x\right] \\
< & \widetilde{c}\left(\varepsilon+\varepsilon^{1 / q}\right)
\end{aligned}
$$

for some constants $c, \widetilde{c}>0$ independent of $n$ and $\varepsilon$. Next, using relation (9),

$$
\begin{aligned}
& \lim _{n \rightarrow \infty} \int_{|x| \leq R^{\varepsilon}} K(x)\left(\left|u_{n}\right|^{q}-\left|u_{0}\right|^{q}\right) d x=0, \\
& \lim _{n \rightarrow \infty} \int_{|x| \leq R^{\varepsilon}} K(x)\left|u_{n}-u_{0}\right|^{q} d x=0 .
\end{aligned}
$$

It follows from relations (10)-(13) that

$$
\limsup _{n \rightarrow \infty}\left|\int_{\mathbb{R}^{N}} K(x)\left(\left|u_{n}\right|^{q}-\left|u_{0}\right|^{q}-\left|u_{n}-u_{0}\right|^{q}\right) d x\right| \leq(q \widetilde{c}+1)\left(\varepsilon+\varepsilon^{1 / q}\right) .
$$

Thanks to $\varepsilon>0$ is arbitrary we conclude that

$$
\lim _{n \rightarrow \infty} \int_{\mathbb{R}^{N}} K(x)\left(\left|u_{n}\right|^{q}-\left|u_{0}\right|^{q}-\left|u_{n}-u_{0}\right|^{q}\right) d x=0
$$

which completes our proof.

Lemma 2.11. Let $\left\{v_{n}\right\}$ be a sequence which is weakly convergent to 0 in $H_{b}\left(\mathbb{R}^{N}\right)$. Then

$$
\begin{aligned}
& \lim _{n \rightarrow \infty}\left[\Upsilon\left(v_{n}\right)-\Upsilon_{\infty}\left(v_{n}\right)\right]=0, \\
& \lim _{n \rightarrow \infty}\left[\left\langle\Upsilon^{\prime}\left(v_{n}\right), v_{n}\right\rangle-\left\langle\Upsilon_{\infty}^{\prime}\left(v_{n}\right), v_{n}\right\rangle\right]=0
\end{aligned}
$$


Proof. By a simple computation we obtain that

$$
\begin{gathered}
\Upsilon\left(v_{n}\right)=\Upsilon_{\infty}\left(v_{n}\right)-\frac{1}{2} \int_{\mathbb{R}^{N}}\left(b_{\infty}-b(x)\right)\left|v_{n}\right|^{2} d x-\frac{1}{q} \int_{\mathbb{R}^{N}}\left(K_{\infty}-K(x)\right)\left|v_{n}\right|^{q} d x, \\
\left.\Upsilon^{\prime}\left(v_{n}\right), v_{n}\right\rangle=\left\langle\Upsilon_{\infty}^{\prime}\left(v_{n}\right), v_{n}\right\rangle-\int_{\mathbb{R}^{N}}\left(b_{\infty}-b(x)\right)\left|v_{n}\right|^{2} d x \\
-\int_{\mathbb{R}^{N}}\left(K_{\infty}-K(x)\right)\left|v_{n}\right|^{q} d x .
\end{gathered}
$$

Let $\varepsilon$ be a positive number. The hypothesis $(K)$ yields that there is $R_{\varepsilon}>0$ such that

$$
\left|K(x)-K_{\infty}\right|=K(x)-K_{\infty}<\varepsilon \text { for a.e. } x \in \mathbb{R}^{N} \text { with }|x|>R_{\varepsilon} .
$$

Applying this fact we get that

$$
\begin{aligned}
\int_{\mathbb{R}^{N}}\left(K(x)-K_{\infty}\right)\left|v_{n}\right|^{q} d x= & \int_{|x| \leq R_{\varepsilon}}\left(K(x)-K_{\infty}\right)\left|v_{n}\right|^{q} d x \\
& +\int_{|x|>R_{\varepsilon}}\left(K(x)-K_{\infty}\right)\left|v_{n}\right|^{q} d x \\
\leq & \left(\|K\|_{\infty}-K_{\infty}\right) \int_{|x| \leq R_{\varepsilon}}\left|v_{n}\right|^{q} d x \\
& +\varepsilon \int_{|x|>R_{\varepsilon}}\left|v_{n}\right|^{q} d x .
\end{aligned}
$$

Due to $v_{n} \rightarrow 0$ in $H_{b}\left(\mathbb{R}^{N}\right)$, it follows by Proposition 2.8 that $\left\{v_{n}\right\}$ is bounded in $L^{q}\left(\mathbb{R}^{N}, \mathbb{C}\right)$. Moreover, using $(9)$ we know that $v_{n} \rightarrow 0$ in $L_{\text {loc }}^{q}\left(\mathbb{R}^{N}, \mathbb{C}\right)$. Then letting $n \rightarrow \infty$ we deduce that

$$
\limsup _{n \rightarrow \infty} \int_{\mathbb{R}^{N}}\left(K(x)-K_{\infty}\right)\left|v_{n}\right|^{q} d x \leq M \varepsilon
$$

for some constant $M>0$ independent of $n$ and $\varepsilon$. It follows that

$$
\lim _{n \rightarrow \infty} \int_{\mathbb{R}^{N}}\left(K(x)-K_{\infty}\right)\left|v_{n}\right|^{q} d x=0 .
$$

To prove (14) and (15) we need only to show that

$$
\lim _{n \rightarrow \infty} \int_{\mathbb{R}^{N}}\left(b_{\infty}-b(x)\right)\left|v_{n}\right|^{2} d x=0 .
$$

For this purpose, notice that for every $R>0$ we can obtain that

$$
\begin{aligned}
\int_{\mathbb{R}^{N}}\left(b_{\infty}-b(x)\right)\left|v_{n}\right|^{2} d x= & \int_{|x| \leq R}\left(b_{\infty}-b(x)\right)\left|v_{n}\right|^{2} d x \\
& +\int_{|x|>R}\left(b_{\infty}-b(x)\right)\left|v_{n}\right|^{2} d x \\
\leq & \left(b_{\infty}-b_{1}\right) \int_{|x| \leq R}\left|v_{n}\right|^{2} d x \\
& +\int_{|x|>R}\left(b_{\infty}-b(x)\right)\left|v_{n}\right|^{2} d x .
\end{aligned}
$$


By $(B)$ we get that, for every $\varepsilon>0$, there exists $R_{\varepsilon}>0$ such that

$$
\left|b_{\infty}-b(x)\right|=b_{\infty}-b(x)<\varepsilon \text { for a.e. } x \in \mathbb{R}^{N} \text { with }|x|>R_{\varepsilon} .
$$

But, it follows from Remark 2.9 that $H_{b}\left(\mathbb{R}^{N}\right)$ is continuously embedded in $L^{2}\left(\mathbb{R}^{N}, \mathbb{C}\right)$. Furthermore, using $(9)$ we see that $v_{n} \rightarrow 0$ in $L_{\text {loc }}^{2}\left(\mathbb{R}^{N}, \mathbb{C}\right)$. Thus, by relations (17) and (18) we deduce that there is a positive number $L$ which is independent of $n$ and $\varepsilon$ such that

$$
\limsup _{n \rightarrow \infty} \int_{\mathbb{R}^{N}}\left(b_{\infty}-b(x)\right)\left|v_{n}\right|^{2} d x \leq L \varepsilon .
$$

Due to $\varepsilon>0$ is a arbitrary, it follows that (16) holds true. This proof is now complete.

Definition 2.12. If $F$ is a $C^{1}$ functional on some Banach space $X$ and $c$ is a real number, we say that a sequence $\left\{u_{n}\right\}$ in $X$ is a $(P S)_{c}$ sequence of $F$ if $F\left(u_{n}\right) \rightarrow c$ and $F^{\prime}\left(u_{n}\right) \rightarrow 0$ in $X^{*}$.

\section{Proof of Theorem 2.4}

We first prove that the weak limit (if this exists) of any $(P S)_{c}$ sequence of the energy functional $J$ is a solution of problem (1).

Lemma 3.1. Let $\left\{u_{n}\right\} \subset H_{A}(\Omega)$ be a $(P S)_{c}$ sequence of $J$ for some $c \in \mathbb{R}$. Assume that $\left\{u_{n}\right\}$ converges weakly to $u_{0}$ in $H_{A}(\Omega)$. Then $J^{\prime}\left(u_{0}\right)=0$, that is, $u_{0}$ is a weak solution of problem (1).

Proof. Consider an arbitrary function $\xi \in C_{0}^{\infty}(\Omega, \mathbb{C})$ and set $\Theta=\operatorname{supp} \xi$. Clearly, the fact that $J^{\prime}\left(u_{n}\right) \rightarrow 0$ in $H_{A}^{-1}(\Omega)$ implies $\left\langle J^{\prime}\left(u_{n}\right), \xi\right\rangle \rightarrow 0$ as $n \rightarrow \infty$, that is,

$$
\lim _{n \rightarrow \infty} \operatorname{Re}\left(\int_{\Theta} \nabla_{A} u_{n} \overline{\nabla_{A} \xi} d x-\int_{\Theta}\left|u_{n}\right|^{q-2} u_{n} \bar{\xi} d x-\lambda \int_{\Theta} f \bar{\xi} d x\right)=0 .
$$

It follows that

$$
\lim _{n \rightarrow \infty} \operatorname{Re} \int_{\Theta} \nabla_{A} u_{n} \overline{\nabla_{A} \xi} d x=\operatorname{Re} \int_{\Theta} \nabla_{A} u_{0} \overline{\nabla_{A} \xi} d x
$$

since $u_{n} \rightarrow u_{0}$ in $H_{A}(\Omega)$. The boundedness of $\left\{u_{n}\right\}$ in $H_{A}(\Omega)$ and the continuous embedding $H_{A}(\Omega) \hookrightarrow L^{q}(\Omega, \mathbb{C})$ imply that $\left\{\left|u_{n}\right|^{q-2} u_{n}\right\}$ is bounded sequence in $L^{q /(q-1)}(\Omega, \mathbb{C})$. Combining this with the convergence (up to a subsequence)

$$
\left|u_{n}\right|^{q-2} u_{n} \rightarrow\left|u_{0}\right|^{q-2} u_{0} \text { a.e. in } \Omega,
$$

we can get (see [7]) that $\left|u_{0}\right|^{q-2} u_{0}$ is the weak limit of the sequence $\left|u_{n}\right|^{q-2} u_{n}$ in $L^{q /(q-1)}(\Omega, \mathbb{C})$. Hence,

$$
\lim _{n \rightarrow \infty} \operatorname{Re} \int_{\Theta}\left|u_{n}\right|^{q-2} u_{n} \bar{\xi} d x=\operatorname{Re} \int_{\Theta}\left|u_{0}\right|^{q-2} u_{0} \bar{\xi} d x .
$$

By relations (19), (20) and (21) we conclude that

$$
\operatorname{Re}\left(\int_{\Theta} \nabla_{A} u_{0} \overline{\nabla_{A} \xi} d x-\int_{\Theta}\left|u_{0}\right|^{q-2} u_{0} \bar{\xi} d x-\lambda \int_{\Theta} f \bar{\xi} d x\right)=0 .
$$


By density, this equality holds for any $\xi \in H_{A}(\Omega)$ which implies that $J^{\prime}\left(u_{0}\right)=0$.

This proof is now complete.

Lemma 3.2. For any $0<\varepsilon<1$ there exist $R=R(\varepsilon)>0$ and $C=C(\varepsilon)>0$ such that for all $f \neq 0$ and $0<\lambda<\frac{C}{\|f\|_{-1}}$, there exists a $(P S)_{c_{0}}$ sequence of $J(u)$ with $c_{0}=c_{0}(R)=\inf _{\bar{B}_{R}} J(u), \bar{B}_{R}=\left\{u \in H_{A}(\Omega):\|u\|_{A} \leq R\right\}$. Furthermore, $c_{0}(R)$ is achieved by some $u_{0} \in H_{A}(\Omega)$ with $J^{\prime}\left(u_{0}\right)=0$.

Proof. Fix $0<\varepsilon<1$. Then for any $u \in H_{A}(\Omega)$, using the Sobolev and Young's inequalities we obtain

$$
\begin{aligned}
J(u) & =\frac{1}{2}\|u\|_{A}^{2}-\frac{1}{q} \int_{\Omega}|u|^{q} d x-\operatorname{Re} \int_{\Omega} \lambda f \bar{u} d x \\
& \geq \frac{1}{2}\|u\|_{A}^{2}-\frac{1}{q}\|u\|_{L^{q}(\Omega, \mathbb{C})}^{q}-\lambda\|u\|_{A}\|f\|_{-1} \\
& \geq \frac{1}{2}\|u\|_{A}^{2}-\frac{1}{q} C_{0}^{q}\|u\|_{A}^{q}-\left(\frac{\varepsilon^{2}}{2}\|u\|_{A}^{2}+\frac{\lambda^{2}}{2 \varepsilon^{2}}\|f\|_{-1}^{2}\right) \\
& =\left(\frac{1}{2}-\frac{\varepsilon^{2}}{2}\right)\|u\|_{A}^{2}-\frac{1}{q} C_{0}^{q}\|u\|_{A}^{q}-\frac{\lambda^{2}}{2 \varepsilon^{2}}\|f\|_{-1}^{2},
\end{aligned}
$$

where $C_{0}>0$ is a positive Sobolev constant given by the continuous embedding $H_{A}(\Omega) \hookrightarrow L^{q}(\Omega, \mathbb{C})$. The above estimate implies the existence of $R=R(\varepsilon)>0$, $C=C(\varepsilon)>0$ and $\delta=\delta(R)>0$ such that $\left.J(u)\right|_{\partial B_{R}} \geq \delta>0$ for all $f \neq 0$ and $0<\lambda<\frac{C}{\|f\|_{-1}}$. For instance, we can take

$$
R(\varepsilon)=\left(\frac{1-\varepsilon^{2}}{C_{0}^{q}}\right)^{1 /(q-2)}, \quad C(\varepsilon)=\sqrt{M} \varepsilon, \quad \delta(R)=\frac{M}{2},
$$

where $M=M(R)=\left(\frac{1}{2}-\frac{1}{q}\right) C_{0}^{q} R^{q}$.

Define $c_{0}=c_{0}(R)=\inf _{\bar{B}_{R}} J(u)$. Since $f \neq 0$ and $\lambda>0$, we have $c_{0}<J(0)=0$. The set $\bar{B}_{R}$ becomes a complete metric space with respect to the distance

$$
\operatorname{dist}(u, v)=\|u-v\|_{A} \quad \text { for any } u, v \in \bar{B}_{R} .
$$

Additionally, $J$ is weakly lower semi-continuous and bounded from below on $\bar{B}_{R}$. Thus, similarly with the proof of Corollary I.5.3 of Struwe [24] (see also [25, Corollary $2.5]$ ), we can deduce that there exists a minimizing sequence $\left\{u_{n}\right\}$ of $J$ with $\left\|u_{n}\right\|_{A}<$ $R$ such that

$$
J\left(u_{n}\right) \rightarrow c_{0} \quad \text { and } \quad J^{\prime}\left(u_{n}\right) \rightarrow 0 \text { in } H_{A}^{-1}(\Omega) .
$$

But the fact that $\left\|u_{n}\right\|_{A}<R$, for fixed $R$, implies that $\left\{u_{n}\right\}$ converges weakly (up to a subsequence) in $H_{A}(\Omega)$. Combining the compact embedding $H_{A}(\Omega) \hookrightarrow$ $L^{q}(\Omega, \mathbb{C})$, relation $(22)$ and Lemma 3.1 we obtain that for some $u_{0} \in H_{A}(\Omega)$

$$
\begin{aligned}
& u_{n} \rightarrow u_{0} \text { in } H_{A}(\Omega), \quad u_{n} \rightarrow u_{0} \text { a.e. in } \Omega, \\
& J^{\prime}\left(u_{0}\right)=0 .
\end{aligned}
$$


Combining (22)-(23) and the weakly lower semi-continuity of $J$, we can deduce that

$$
c_{0}=\liminf _{n \rightarrow \infty} J\left(u_{n}\right) \geq J\left(u_{0}\right) .
$$

Due to $u_{0} \in \bar{B}_{R}$, we see that $J\left(u_{0}\right)=c_{0}$.

This proof is now complete.

\subsection{The second solution of Theorem 2.4}

Now we show that the functional $J$ satisfies the mountain pass geometry (see [25]).

Lemma 3.3. For any fixed $0<\varepsilon_{1}<1$ and $f \neq 0$, the functional $J$ satisfies the following properties:

(i) there are $R_{1}>0, C_{1}=C_{1}\left(\varepsilon_{1}\right)=C_{1}\left(R_{1}\right)>0$ and $\delta_{R_{1}}>0$ such that for all $0<\lambda<\frac{C_{1}}{\|f\|_{-1}}$ we have $\left.J\right|_{\partial B_{R_{1}}} \geq \delta_{R_{1}}$

(ii) there is $e \in H_{A}(\Omega)$ with $\|e\|_{A}>R_{1}$ such that $J(e)<0$.

Proof. (i) Using the conclusion and proof of Lemma 3.2, we can easily get this result.

(ii) For each $0 \neq u \in H_{A}(\Omega)$ and $t>0$, one has

$$
J(t u) \leq \frac{t^{2}}{2}\|u\|_{A}^{2}-\frac{t^{q}}{q} \int_{\Omega}|u|^{q} d x+\lambda t\|f\|_{-1}\|u\|_{A} .
$$

Since $q>2$, this relation shows that for all $\lambda \in\left(0, \frac{C_{1}}{\|f\|_{-1}}\right)$ we can find $t_{\lambda}>0$ such that $J\left(t_{\lambda} u\right)<0$. So, we get the conclusion.

This proof is now complete.

Lemma 3.4. For any $c \in \mathbb{R}$ and $f \neq 0$ with $\lambda \in\left(0, \frac{C_{1}}{\|f\|_{-1}}\right)\left(C_{1}\right.$ is given in Lemma 3.3), $J$ satisfies $(P S)_{c}$ condition, namely any $(P S)_{c}$ sequence of $J$ has a convergent subsequence in $H_{A}(\Omega)$.

Proof. Let $\left\{u_{n}\right\} \subset H_{A}(\Omega)$ be a $(P S)_{c}$ sequence of $J$. It follows that

$$
J\left(u_{n}\right)=c+o(1) \text { and }\left\|J^{\prime}\left(u_{n}\right)\right\|_{-1}=o(1) \text { as } n \rightarrow \infty .
$$

So, we have

$$
\begin{aligned}
q J\left(u_{n}\right)-\left\langle J\left(u_{n}\right), u_{n}\right\rangle & =\frac{q-2}{2}\left\|u_{n}\right\|_{A}^{2}-(q-1) \operatorname{Re} \int_{\Omega} \lambda f \overline{u_{n}} d x \\
& =O(1)+o(1)\|u\|_{A} \text { as } n \rightarrow \infty
\end{aligned}
$$

Note that $2<q<2^{*}$ and $\lambda \in\left(0, \frac{C_{1}}{\|f\|_{-1}}\right)$, for $n$ big enough we can find that there exists a constant $C>0$ such that

$$
c+1+\left\|u_{n}\right\|_{A} \geq \frac{q-2}{2}\left\|u_{n}\right\|_{A}^{2}-C\left\|u_{n}\right\|_{A} .
$$

It follows immediately that that $\left\{u_{n}\right\}$ is bounded in $H_{A}(\Omega)$. Thus, up to a subsequence, we may assume that there exists $u^{\prime} \in H_{A}(\Omega)$ such that

$$
\begin{aligned}
& u_{n} \rightarrow u^{\prime} \text { in } H_{A}(\Omega), \\
& u_{n} \rightarrow u^{\prime} \text { in } L^{\mu}(\Omega) \text { for all } \mu \in\left[1,2^{*}\right) .
\end{aligned}
$$


Using this fact and Hölder's inequality, it is easy to see that

$$
\begin{aligned}
\lim _{n \rightarrow \infty}\left\|u_{n}-u^{\prime}\right\|_{A}^{2}= & \lim _{n \rightarrow \infty}\left\langle J^{\prime}\left(u_{n}\right)-J^{\prime}\left(u^{\prime}\right), u_{n}-u^{\prime}\right\rangle \\
& +\lim _{n \rightarrow \infty} \operatorname{Re} \int_{\Omega}\left(\left|u_{n}\right|^{q-2} u_{n}-\left|u^{\prime}\right|^{q-2} u^{\prime}\right) \overline{\left(u_{n}-u^{\prime}\right)} d x \\
& +\lim _{n \rightarrow \infty} \operatorname{Re} \int_{\Omega} \lambda f \overline{\left(u_{n}-u\right)} d x \\
= & 0,
\end{aligned}
$$

that is, $\left\|u_{n}-u^{\prime}\right\|_{A} \rightarrow 0$ as $n \rightarrow \infty$.

This proof is now complete.

On the other hand, it is easy to see that $J(0)=0$. Combining the Mountain Pass Theorem [21, p. 4], Lemma 3.3 and Lemma 3.4 we obtain

$$
J\left(u^{\prime}\right)=c_{1}>0 \text { and } J^{\prime}\left(u^{\prime}\right)=0,
$$

that is, $u$ is a weak solution of problem (1), where

$$
c_{1}=\inf _{\gamma \in \Gamma} \max _{t \in[0,1]} J(\gamma(t))
$$

and

$$
\Gamma=\left\{\gamma \in C\left([0,1], H_{A}(\Omega)\right): \gamma(0)=0 \text { and } \gamma(1)=e\right\}
$$

\subsection{Proof of Theorem 2.4 completed}

Consider $R_{1}>0, C_{1}=C_{1}\left(R_{1}\right)>0$ and $\delta_{R_{1}}>0$ given by Lemma 3.3. Thus, in light of its proof, we are able to obtain that for all $f \neq 0$ and $0<\lambda<\frac{C_{1}}{\|f\|_{-1}}$ the conclusion of Lemma 3.2 also holds true. Hence, we get the existence of a solution $u_{0} \in H_{A}(\Omega)$ of problem $(1)$ such that $J\left(u_{0}\right)=c_{0}<0$.

Clearly, $u_{0} \neq u^{\prime}$. This proof of Theorem 2.4 is now complete.

\section{Proof of Theorem 2.5}

The energy functional associated to problem (2) is $\mathcal{J}: H_{A}(\Omega) \rightarrow \mathbb{R}$ defined by

$$
\mathcal{J}(u)=\frac{1}{2} \int_{\Omega}\left|\nabla_{A} u\right|^{2} d x-\frac{1}{2^{*}} \int_{\Omega}|u|^{2^{*}} d x-\frac{\lambda}{q} \int_{\Omega}|u|^{q} d x \text { for all } u \in H_{A}(\Omega) .
$$

Let $S$ denote the best constant of the continuous embedding of $H_{A}(\Omega)$ into $L^{2^{*}}(\Omega, \mathbb{C})$, that is,

$$
S:=\inf _{u \in H_{A}(\Omega) \backslash\{0\}} \frac{\int_{\Omega}\left|\nabla_{A} u\right|^{2} d x}{\left(\int_{\Omega}|u|^{2^{*}} d x\right)^{2 / 2^{*}}} .
$$

Lemma 4.1. Every $(P S)_{c}$ sequence of $\mathcal{J}$ is bounded in $H_{A}(\Omega)$. 
Proof. Let $\left\{u_{n}\right\} \subset H_{A}(\Omega)$ be a $(P S)_{c}$ sequence of $\mathcal{J}$. It follows that

$$
\mathcal{J}\left(u_{n}\right)=c+o(1) \text { and }\left\|\mathcal{J}^{\prime}\left(u_{n}\right)\right\|_{-1}=o(1) \text { as } n \rightarrow \infty .
$$

It follows that

$$
\begin{aligned}
2^{*} \mathcal{J}\left(u_{n}\right)-\left\langle\mathcal{J}^{\prime}\left(u_{n}\right), u_{n}\right\rangle & =\left(2^{*}-1\right)\left\|u_{n}\right\|_{A}^{2}-\lambda\left(\frac{2^{*}}{q}-1\right) \int_{\Omega}\left|u_{n}\right|^{q} d x \\
& =O(1)+o(1)\left\|u_{n}\right\|_{A} \quad \text { as } n \rightarrow \infty .
\end{aligned}
$$

First of all, note that $1<q<2$. Fix $\varepsilon>0$ small enough. Combining the Hölder's and Young's inequalities we obtain for all $u \in H_{A}(\Omega)$

$$
\begin{aligned}
\frac{\lambda}{q} \int_{\Omega}|u|^{q} d x & \leq \frac{\lambda}{q} S^{-q / 2}\|u\|_{A}^{q}\left(\int_{\Omega} 1^{2^{*} /\left(2^{*}-q\right)} d x\right)^{\left(2^{*}-q\right) / 2^{*}} \\
& \leq C(\varepsilon) \lambda^{2 /(2-q)}+\frac{q}{2} \varepsilon\|u\|_{A}^{2}
\end{aligned}
$$

where

$$
C(\varepsilon)=\frac{2-q}{2} q^{\frac{2}{q-2}}(\varepsilon S)^{q /(q-2)}|\Omega|^{[N(2-q)+2 q] /[N(2-q)]}>0 .
$$

Combining relations (24) and (25) we have

$$
\left(2^{*}-1-\frac{2^{*} q}{2} \varepsilon+\frac{q}{2} \varepsilon\right)\left\|u_{n}\right\|_{A}^{2}-\left(2^{*}-q\right) C(\varepsilon) \lambda^{2 /(2-q)} \leq O(1)+o(1)\left\|u_{n}\right\|_{A} .
$$

Since $\varepsilon>0$ can be chosen sufficiently small, we deduce that $\left\{u_{n}\right\} \subset H_{A}(\Omega)$ is bounded.

Lemma 4.2. There exists a real number $c^{*}$ such that $\mathcal{J}$ satisfies the $(P S)_{c}$ condition for all $c<c^{*}$.

Proof. Fix $c \in \mathbb{R}$. Let $\left\{u_{n}\right\} \subset H_{A}(\Omega)$ be an arbitrary $(P S)_{c}$ sequence of $\mathcal{J}$. By Lemma 4.1, $\left\{u_{n}\right\}$ is bounded. Thus, up to a subsequence

$$
\begin{gathered}
u_{n} \rightarrow u_{0} \text { in } H_{A}(\Omega), \\
u_{n} \rightarrow u_{0} \text { in } L^{q}(\Omega, \mathbb{C}),
\end{gathered}
$$

and there exists $\psi \in L^{2^{*}-1}(\Omega, \mathbb{R})$ such that

$$
u_{n} \rightarrow u_{0} \text { a.e. on } \Omega \text { and }\left|u_{n}\right| \leq \psi \text { for all } n \text {, a.e. on } \Omega \text {. }
$$

Taking $n \rightarrow \infty$, it follows that for all $v \in H_{A}(\Omega)$

$$
\begin{aligned}
& \operatorname{Re} \int_{\Omega} \nabla_{A} u_{n} \overline{\nabla_{A} v} d x \rightarrow \operatorname{Re} \int_{\Omega} \nabla_{A} u_{0} \overline{\nabla_{A} v} d x \\
& \operatorname{Re} \int_{\Omega}\left|u_{n}\right|^{q-2} u_{n} \bar{v} d x \rightarrow \operatorname{Re} \int_{\Omega}\left|u_{0}\right|^{q-2} u_{0} \bar{v} d x,
\end{aligned}
$$

and

$$
\operatorname{Re} \int_{\Omega}\left|u_{n}\right|^{2^{*}-2} u_{n} \bar{v} d x \rightarrow \operatorname{Re} \int_{\Omega}\left|u_{0}\right|^{2^{*}-2} u_{0} \bar{v} d x
$$

Since $\left\|\mathcal{J}^{\prime}\left(u_{n}\right)\right\|_{-1}=o(1)$ as $n \rightarrow \infty$, these relations show that

$$
\left\langle\mathcal{J}^{\prime}\left(u_{0}\right), v\right\rangle=0 \text { for all } v \in H_{A}(\Omega) .
$$


Therefore

$$
\begin{aligned}
\mathcal{J}\left(u_{0}\right)= & \mathcal{J}\left(u_{0}\right)-\frac{1}{2^{*}}\left\langle\mathcal{J}^{\prime}\left(u_{0}\right), u_{0}\right\rangle \\
\geq & \frac{1}{2^{*}}\left(\frac{2}{N-2}-\frac{N(2-q)+2 q}{N-2} \varepsilon\right)\left\|u_{0}\right\|_{A}^{2} \\
& -\frac{1}{2^{*}} \frac{N(2-q)+2 q}{N-2} C(\varepsilon) \lambda^{2 /(2-q)} .
\end{aligned}
$$

Choose $\varepsilon=\frac{2}{(2-q) N+2 q}$. Then relation (26) yields the following lower estimate

$$
\mathcal{J}\left(u_{0}\right) \geq-A(q, N) \lambda^{2 /(2-q)},
$$

where $A(q, N)$ is a positive constant.

Fix $\lambda>0$ such that

$$
c^{*}:=\frac{S^{N / 2}}{N}-A(q, N) \lambda^{2 /(2-q)}>0 .
$$

We already know that

$$
\int_{\Omega}\left|u_{n}\right|^{q} d x=\int_{\Omega}\left|u_{0}\right|^{q} d x+o(1) \text { as } n \rightarrow \infty .
$$

Next, the Brezis-Lieb theorem implies

$$
\left\|u_{n}\right\|_{A}^{2}=\left\|u_{n}-u_{0}\right\|_{A}^{2}+\left\|u_{0}\right\|_{A}^{2}+o(1) \text { as } n \rightarrow \infty
$$

and

$$
\int_{\Omega}\left|u_{n}\right|^{2^{*}} d x=\int_{\Omega}\left|u_{n}-u_{0}\right|^{2^{*}} d x+\int_{\Omega}\left|u_{0}\right|^{2^{*}} d x+o(1) \text { as } n \rightarrow \infty .
$$

Since $\left\{u_{n}\right\}$ is a $(P S)_{c}$ sequence, relations (29), (30) and (31) imply that

$$
\begin{aligned}
\mathcal{J}\left(u_{n}\right)-\mathcal{J}\left(u_{0}\right) & =\frac{1}{2}\left\|u_{n}-u_{0}\right\|_{A}^{2}-\frac{1}{2^{*}} \int_{\Omega}\left|u_{n}-u_{0}\right|^{2^{*}} d x+o(1) \\
& =c-\mathcal{J}\left(u_{0}\right)+o(1) \text { as } n \rightarrow \infty .
\end{aligned}
$$

Next, using the fact that $\left\|\mathcal{J}^{\prime}\left(u_{n}\right)\right\|_{-1}=o(1)$ as $n \rightarrow \infty$ in conjunction with $\left\langle\mathcal{J}^{\prime}\left(u_{0}\right), u_{0}\right\rangle=0$ we obtain

$$
\left\|u_{n}-u_{0}\right\|_{A}^{2}-\int_{\Omega}\left|u_{n}-u_{0}\right|^{2^{*}} d x=o(1) \text { as } n \rightarrow \infty .
$$

Combining relations (32) and (33) we deduce that there exists $\ell \geq 0$ such that

$$
\left\|u_{n}-u_{0}\right\|_{A}=\ell+o(1) \text { as } n \rightarrow \infty \text {. }
$$

We claim that if $c<c^{*}$ (where $c^{*}$ is defined in (28)) then $\ell=0$, which implies that the sequence $\left\{u_{n}\right\}$ is convergent (up to a subsequence) to $u_{0}$ in $H_{A}(\Omega)$.

Fix $c<c^{*}$ and assume that $\ell>0$. Then relation (33) implies that

$$
\int_{\Omega}\left|u_{n}-u_{0}\right|^{2^{*}} d x \rightarrow \ell^{2} \text { as } n \rightarrow \infty .
$$

But

$$
\left\|u_{n}-u_{0}\right\|_{A}^{2^{*}} \geq S^{2^{*} / 2} \int_{\Omega}\left|u_{n}-u_{0}\right|^{2^{*}} d x .
$$


Thus, as $n \rightarrow \infty$, we obtain

$$
\ell^{2^{*}} \geq S^{2^{*} / 2} \ell^{2}
$$

hence $\ell \geq S^{N / 4}$. It follows that

$$
c^{*}=\frac{S^{N / 2}}{N}-A(q, N) \lambda^{2 /(2-q)} \leq \frac{\ell^{2}}{N}+\mathcal{J}\left(u_{0}\right)=c,
$$

which contradicts the choice of $c$. This proves our claim and the proof is now complete.

By relation (25) we have for all $u \in H_{A}(\Omega)$

$$
\mathcal{J}(u) \geq\left(\frac{1}{2}-\varepsilon\right)\|u\|_{A}^{2}-C(\varepsilon) \lambda^{2 /(2-q)}-\frac{1}{2^{*} S^{N /(N-2)}}\|u\|_{A}^{2^{*}} .
$$

Taking $\varepsilon \in(0,1 / 2)$, we can choose two positive numbers $\lambda^{*}$ and $r$ so that both relation $(28)$ and

$$
\mathcal{J}(u)>0 \text { for all } \lambda \in\left(0, \lambda^{*}\right) \text { and all } u \in H_{A}(\Omega) \text { with }\|u\|_{A}=r
$$

hold. This property establishes the existence of a "mountain" near the origin and for small perturbations (that is, small positive values of the parameter).

Next, we prove the existence of a "valley". For this purpose, let $\phi_{1}$ be a positive element in $H_{A}(\Omega)$. It follows that for all $t>0$

$$
\mathcal{J}\left(t \phi_{1}\right) \leq \frac{t^{2}}{2}\left\|\phi_{1}\right\|_{A}^{2}-\frac{t^{2^{*}}}{2^{*}} \int_{\Omega} \phi_{1}^{2^{*}} d x-\frac{\lambda t^{q}}{q} \int_{\Omega} \phi_{1}^{q} d x .
$$

This relation shows that for all $\lambda \in\left(0, \lambda^{*}\right)$ we can find $t_{\lambda}>0$ such that $\mathcal{J}\left(t_{\lambda} \phi_{1}\right)<0$, hence

$$
c_{\lambda}:=\inf \left\{\mathcal{J}(u) ;\|u\|_{A} \leq r\right\}<0<\inf \left\{\mathcal{J}(u) ;\|u\|_{A}=r\right\}
$$

Therefore, similarly with the proof of Corollary I.5.3 of Struwe [24] (see also [25, Corollary 2.5]), we can deduce that there exists a minimizing sequence $\left\{u_{n}\right\}$ of $\mathcal{J}$ with $\left\|u_{n}\right\|_{A}<r$ such that

$$
\mathcal{J}\left(u_{n}\right) \rightarrow c_{\lambda} \text { and }\left\|\mathcal{J}^{\prime}\left(u_{n}\right)\right\|_{-1} \rightarrow 0
$$

Combining Lemma 4.2 with the fact that $c_{\lambda}<0$, we deduce that this minimizing sequence is relatively compact in $H_{A}(\Omega)$. It follows that its limit is a solution of problem (2). This solution is nontrivial, since $c_{\lambda}<0$.

The proof of Theorem 2.5 is now complete.

\section{Proof of Theorem 2.6}

We start by proving that the weak limit (if this exists) of any $(P S)_{c}$ sequence of $\Psi$ is a solution of problem (3).

Lemma 5.1. Let $\left\{u_{n}\right\} \subset H_{b}\left(\mathbb{R}^{N}\right)$ be a $(P S)_{c}$ sequence of $\Psi$ for some $c \in \mathbb{R}$. Assume that $\left\{u_{n}\right\}$ converges weakly to $u_{0}^{\prime}$ in $H_{b}\left(\mathbb{R}^{N}\right)$. Then $\Psi^{\prime}\left(u_{0}^{\prime}\right)=0$, that is, $u_{0}^{\prime}$ is a weak solution of problem (3). 
Proof. Consider an arbitrary function $\xi^{\prime} \in C_{0}^{\infty}\left(\mathbb{R}^{N}, \mathbb{C}\right)$ and set $\Theta=\operatorname{supp} \xi^{\prime}$. Obviously $\Psi^{\prime}\left(u_{n}\right) \rightarrow 0$ in $H_{b}^{-1}\left(\mathbb{R}^{N}\right)$ implies $\left\langle\Psi^{\prime}\left(u_{n}\right), \xi^{\prime}\right\rangle \rightarrow 0$ as $n \rightarrow \infty$, that is,

$$
\begin{aligned}
\lim _{n \rightarrow \infty} \operatorname{Re} & \left(\int_{\Theta}\left(\nabla_{A} u_{n} \overline{\nabla_{A} \xi^{\prime}}+b(x) u_{n} \overline{\xi^{\prime}}\right) d x\right. \\
& \left.-\int_{\Theta} K(x)\left|u_{n}\right|^{q-2} u_{n} \overline{\xi^{\prime}} d x-\int_{\Theta} g(x) \overline{\xi^{\prime}} d x\right)=0 .
\end{aligned}
$$

Since $u_{n} \rightarrow u_{0}^{\prime}$ in $H_{b}\left(\mathbb{R}^{N}\right)$ it follows that

$$
\begin{aligned}
\lim _{n \rightarrow \infty} \operatorname{Re} \int_{\Theta}\left(\nabla_{A} u_{n} \overline{\nabla_{A} \xi^{\prime}}+b(x) u_{n} \overline{\xi^{\prime}}\right) d x \\
=\operatorname{Re} \int_{\Theta}\left(\nabla_{A} u_{0}^{\prime} \overline{\nabla_{A} \xi^{\prime}}+b(x) u_{0}^{\prime} \overline{\xi^{\prime}}\right) d x .
\end{aligned}
$$

From the boundedness of $\left\{u_{n}\right\}$ in $H_{b}\left(\mathbb{R}^{N}\right)$ and Proposition 2.8 we know that $\left\{\left|u_{n}\right|^{q-2} u_{n}\right\}$ is bounded sequence in $L^{q /(q-1)}\left(\mathbb{R}^{N}, \mathbb{C}\right)$. Combining this with the convergence (which is a consequence of (9))

$$
\left|u_{n}\right|^{q-2} u_{n} \rightarrow\left|u_{0}^{\prime}\right|^{q-2} u_{0}^{\prime} \text { a.e. in } \mathbb{R}^{N}
$$

we conclude (see [7]) that $\left|u_{0}^{\prime}\right|^{q-2} u_{0}^{\prime}$ is the weak limit of the sequence $\left|u_{n}\right|^{q-2} u_{n}$ in $L^{q /(q-1)}\left(\mathbb{R}^{N}, \mathbb{C}\right)$. So

$$
\lim _{n \rightarrow \infty} \operatorname{Re} \int_{\Theta} K(x)\left|u_{n}\right|^{q-2} u_{n} \overline{\xi^{\prime}} d x=\operatorname{Re} \int_{\Theta} K(x)\left|u_{0}^{\prime}\right|^{q-2} u_{0}^{\prime} \overline{\xi^{\prime}} d x .
$$

From (35), (36) and (37) we deduce that

$$
\begin{aligned}
& \operatorname{Re}\left(\int_{\Theta}\left(\nabla_{A} u_{0}^{\prime} \overline{\nabla_{A} \xi^{\prime}}+b(x) u_{0}^{\prime} \overline{\xi^{\prime}}\right) d x\right. \\
& \left.\quad-\int_{\Theta} K(x)\left|u_{0}^{\prime}\right|^{q-2} u_{0}^{\prime} \overline{\xi^{\prime}} d x-\int_{\Theta} g(x) \overline{\xi^{\prime}} d x\right)=0 .
\end{aligned}
$$

By density, this equality holds for any $\xi^{\prime} \in H_{b}\left(\mathbb{R}^{N}\right)$ which means that $\Psi^{\prime}\left(u_{0}\right)=0$. This concludes our proof.

Lemma 5.2. For any $0<\varepsilon<1$ there exist $R^{\prime}=R^{\prime}(\varepsilon)>0$ and $C^{\prime}=C^{\prime}(\varepsilon)>0$ such that for all $g \not \equiv 0$ with $\|g\|_{b,-1} \leq C^{\prime}$, there exists a $(P S)_{c_{0}^{\prime}}$ sequence of $\Psi(u)$ with $c_{0}^{\prime}=c_{0}^{\prime}\left(R^{\prime}\right)=\inf _{\bar{B}_{R^{\prime}}} \Psi(u), \bar{B}_{R^{\prime}}=\left\{u \in H_{b}\left(\mathbb{R}^{N}\right):\|u\|_{b} \leq R^{\prime}\right\}$. Furthermore, $c_{0}^{\prime}\left(R^{\prime}\right)$ is achieved by some $u_{0}^{\prime} \in H_{b}\left(\mathbb{R}^{N}\right)$ with $\Psi^{\prime}\left(u_{0}^{\prime}\right)=0$.

Proof. Fix $0<\varepsilon<1$. Then for any $u \in H_{b}\left(\mathbb{R}^{N}\right)$, by $(K)$ and Young's inequality we have

$$
\begin{aligned}
\Psi(u) & =\frac{1}{2}\|u\|_{b}^{2}-\frac{1}{q} \int_{\mathbb{R}^{N}} K(x)|u|^{q} d x-\operatorname{Re} \int_{\mathbb{R}^{N}} g(x) \bar{u} d x \\
& \geq \frac{1}{2}\|u\|_{b}^{2}-\frac{\|K\|_{\infty}}{q}\|u\|_{L^{q}\left(\mathbb{R}^{N}, \mathbb{C}\right)}^{q}-\|u\|_{b}\|g\|_{b,-1} \\
& \geq \frac{1}{2}\|u\|_{b}^{2}-\frac{\|K\|_{\infty}}{q} C_{0}^{q}\|u\|_{b}^{q}-\left(\frac{\varepsilon^{2}}{2}\|u\|_{b}^{2}+\frac{1}{2 \varepsilon^{2}}\|g\|_{b,-1}^{2}\right)
\end{aligned}
$$




$$
=\left(\frac{1}{2}-\frac{\varepsilon^{2}}{2}\right)\|u\|_{b}^{2}-\frac{\|K\|_{\infty}}{q} M_{q}^{q}\|u\|_{b}^{q}-\frac{1}{2 \varepsilon^{2}}\|g\|_{b,-1}^{2},
$$

where $M_{q}>0$ is a positive constant given by Proposition 2.8. The above estimate states the existence of $R^{\prime}=R^{\prime}(\varepsilon)>0, C^{\prime}=C^{\prime}(\varepsilon)>0$ and $\delta^{\prime}=\delta\left(R^{\prime}\right)>0$ such that $\left.\Psi(u)\right|_{\partial B_{R^{\prime}}} \geq \delta^{\prime}>0$ for all $g \not \equiv 0$ with $\|g\|_{b,-1} \leq C^{\prime}$. For example, we can choose

$$
R^{\prime}(\varepsilon)=\left(\frac{1-\varepsilon^{2}}{\|K\|_{\infty} M_{q}^{q}}\right)^{1 /(q-2)}, \quad C^{\prime}(\varepsilon)=\sqrt{M^{\prime}} \varepsilon, \quad \delta\left(R^{\prime}\right)=\frac{M^{\prime}}{2},
$$

where $M^{\prime}=M^{\prime}\left(R^{\prime}\right)=\left(\frac{1}{2}-\frac{1}{q}\right)\|K\|_{\infty} M_{q}^{q} R^{\prime q}$. Let us define $c_{0}^{\prime}=c_{0}^{\prime}\left(R^{\prime}\right)=\inf _{\bar{B}_{R^{\prime}}} \Psi(u)$. Since $g \not \equiv 0, c_{0}^{\prime}<J(0)=0$. The set $\bar{B}_{R^{\prime}}$ becomes a complete metric space with respect to the distance

$$
\operatorname{dist}(u, v)=\|u-v\|_{b} \quad \text { for any } u, v \in \bar{B}_{R^{\prime}}
$$

On the other hand, $\Psi$ is weakly lower semi-continuous and bounded from below on $\bar{B}_{R^{\prime}}$. So, similarly with the proof of Corollary I.5.3 of Struwe [24] (see also [25, Corollary 2.5]), we can deduce that there exists a minimizing sequence $\left\{u_{n}\right\}$ of $\Psi$ with $\left\|u_{n}\right\|_{b}<R^{\prime}$ such that

$$
\Psi\left(u_{n}\right) \rightarrow c_{0}^{\prime} \quad \text { and } \quad \Psi^{\prime}\left(u_{n}\right) \rightarrow 0 \text { in } H_{b}^{-1}\left(\mathbb{R}^{N}\right) .
$$

But $\left\|u_{n}\right\|_{b}<R^{\prime}$, for the fixed $R^{\prime}$, we shows that $\left\{u_{n}\right\}$ converges weakly (up to a subsequence) in $H_{b}\left(\mathbb{R}^{N}\right)$. Therefore, (9), (38) and Lemma 5.1 imply that, for some $u_{0}^{\prime} \in H_{b}\left(\mathbb{R}^{N}\right)$

$$
\begin{aligned}
& u_{n} \rightarrow u_{0}^{\prime} \text { in } H_{b}\left(\mathbb{R}^{N}\right), \quad u_{n} \rightarrow u_{0}^{\prime} \text { a.e. in } \mathbb{R}^{N}, \\
& \Psi^{\prime}\left(u_{0}^{\prime}\right)=0 .
\end{aligned}
$$

We prove that $\Psi\left(u_{0}^{\prime}\right)=c_{0}^{\prime}$. By (38) and (39) we have

$$
\begin{aligned}
o(1) & =\left\langle\Psi^{\prime}\left(u_{n}\right), u_{n}\right\rangle \\
& =\operatorname{Re}\left(\int_{\mathbb{R}^{N}}\left(\left|\nabla_{A} u_{n}\right|^{2}+b(x)\left|u_{n}\right|^{2}\right) d x-\left.\int_{\mathbb{R}^{N}} K(x) u_{n}\right|^{q} d x-\int_{\mathbb{R}^{N}} g(x) \overline{u_{n}} d x\right) .
\end{aligned}
$$

Therefore

$$
\Psi\left(u_{n}\right)=\left(\frac{1}{2}-\frac{1}{q}\right) \int_{\mathbb{R}^{N}} K(x)\left|u_{n}\right|^{q} d x-\frac{1}{2} \operatorname{Re} \int_{\mathbb{R}^{N}} g(x) \overline{u_{n}} d x+o(1) .
$$

By (38)-(40) and Fatou's lemma we have

$$
\begin{aligned}
c_{0}^{\prime} & =\liminf _{n \rightarrow \infty} \Psi\left(u_{n}\right) \\
& \geq\left(\frac{1}{2}-\frac{1}{q}\right) \int_{\mathbb{R}^{N}} K(x)\left|u_{0}^{\prime}\right|^{q} d x-\frac{1}{2} \operatorname{Re} \int_{\mathbb{R}^{N}} g(x) \overline{u_{0}^{\prime}} d x \\
& =\Psi\left(u_{0}^{\prime}\right) .
\end{aligned}
$$

Since $u_{0}^{\prime} \in \bar{B}_{R^{\prime}}$, it follows that $\Psi\left(u_{0}^{\prime}\right)=c_{0}^{\prime}$.

This proof is now complete. 


\subsection{A priori estimate for the second solution of Theorem 2.6}

Firstly, we consider the following constrained minimization problem

$$
m_{\infty}=\inf \left\{\int_{\mathbb{R}^{N}}\left(\left|\nabla_{A} u\right|^{2}+b_{\infty}|u|^{2}\right) d x: u \in H_{b_{\infty}}\left(\mathbb{R}^{N}\right), \int_{\mathbb{R}^{N}} K_{\infty}|u|^{q} d x=1\right\} .
$$

By virtue of $(A)$ we can define a different "translation" $T: H_{A}\left(\mathbb{R}^{N}\right) \times \mathbb{Z}^{N} \rightarrow$ $H_{A}\left(\mathbb{R}^{N}\right)$ by letting $\left(T_{y} u\right)(x):=u(x+y) e^{-i \varphi_{y}(x)}$. Note that in general $T_{y_{1}+y_{2}} \neq$ $T_{y_{2}} T_{y_{1}}$, hence $T$ is not a group action of $\mathbb{Z}^{N}$. That the operator $T$ is well-defined is a consequence of the following property.

Lemma 5.3. Let $u \in H_{A}\left(\mathbb{R}^{N}\right), y \in \mathbb{Z}^{N}$ and $v:=T_{y} u$. Then

$$
v \in H_{A}\left(\mathbb{R}^{N}\right) \text { and } \int_{\mathbb{R}^{N}}\left|\nabla_{A} v\right|^{2} d x=\int_{\mathbb{R}^{N}}\left|\nabla_{A} u\right|^{2} d x .
$$

In particular, for each $y \in \mathbb{Z}^{N}$ the operator $T$ is an isometry.

Proof. Similarly with the proof of Lemma 4.1 of Arioli and Szulkin [4], we can get the conclusion. For the convenience of readers, we give the details. Indeed, from condition $(A)$, it follows that

$$
\begin{aligned}
\nabla_{A} v(x) & =-i \nabla\left(u(x+y) e^{-i \varphi_{y}(x)}\right)-A(x) u(x+y) e^{-i \varphi_{y}(x)} \\
& =-i\left[\nabla u(x+y)-i u(x+y) \nabla \varphi_{y}(x)-i A(x) u(x+y)\right] e^{-i \varphi_{y}(x)} \\
& =-i[\nabla u(x+y)-i A(x+y) u(x+y)] e^{-i \varphi_{y}(x)} \\
& =[-i \nabla u(x+y)-A(x+y) u(x+y)] e^{-i \varphi_{y}(x)},
\end{aligned}
$$

thus $\int_{\mathbb{R}^{N}}\left|\nabla_{A} v\right|^{2} d x=\int_{\mathbb{R}^{N}}\left|\nabla_{A} u\right|^{2} d x$. Furthermore, $\int_{\mathbb{R}^{N}}|v|^{2} d x=\int_{\mathbb{R}^{N}}|u|^{2} d x$ and $\int_{\mathbb{R}^{N}}|v|^{q} d x=\int_{\mathbb{R}^{N}}|u|^{q} d x$, hence the conclusion.

Let $s \in \mathbb{R},[s]$ is defined as the largest integer not exceeding $s$, that is, $[s] \leq s<$ $[s]+1$. For $y=\left(y^{1}, y^{2}, \ldots, y^{N}\right) \in \mathbb{R}^{N}$, we use $[y]$ to denote $[y]=\left(\left[y^{1}\right],\left[y^{2}\right], \ldots,\left[y^{N}\right]\right)$. It is easy to see that $|[y]-y| \leq \sqrt{N}$.

Lemma 5.4. Assume that the hypotheses $(A),(B)$ and $(K)$ are fulfilled. Let $\left\{u_{n}\right\} \subset$ $H_{b_{\infty}}\left(\mathbb{R}^{N}\right)$ be a minimizing sequence for problem $(41)$. There is a $v \in H_{b_{\infty}}\left(\mathbb{R}^{N}\right)$ such that

$$
m_{\infty}=\int_{\mathbb{R}^{N}}\left(\left|\nabla_{A} v\right|^{2}+b_{\infty}|v|^{2}\right) d x \text { and } \int_{\mathbb{R}^{N}} K_{\infty}|v|^{q} d x=1 .
$$

Proof. Combining conditions $(B),(K)$ and Proposition 2.8 we see that $m_{\infty}>0$. Due to $\int_{\mathbb{R}^{N}} K_{\infty}\left|u_{n}\right|^{q} d x=1$ and $K_{\infty}>0$, Lemma 1.21 of [25] yields

$$
d:=\limsup _{n \rightarrow \infty} \sup _{y \in \mathbb{R}^{N}} \int_{B(y, 1)}\left|u_{n}\right|^{2} d x>0
$$


Going if necessary to a subsequence, we may assume the existence of $\left\{y_{n}\right\} \subset \mathbb{R}^{N}$ such that

$$
\int_{B\left(y_{n}, 1\right)}\left|u_{n}\right|^{2} d x>\frac{d}{2} .
$$

So, using the above inequality we can easily obtain

$$
\int_{B\left(\left[y_{n}\right], \sqrt{N}+1\right)}\left|u_{n}\right|^{2} d x>\frac{d}{2} .
$$

Let us define $v_{n}:=T_{\left[y_{n}\right]} u_{n}$. Applying Lemma 5.3 we see that

$$
\int_{\mathbb{R}^{N}}\left|v_{n}\right|^{q} d x=1 / K_{\infty},\left\|v_{n}\right\|_{b_{\infty}}^{2} \rightarrow m_{\infty}
$$

and

$$
\int_{B(0, \sqrt{N}+1)}\left|v_{n}\right|^{2} d x>\frac{d}{2} .
$$

Since $v_{n}$ is bounded in $H_{b_{\infty}}\left(\mathbb{R}^{N}\right)$, we may assume, going if necessary to a subsequence

$$
\begin{aligned}
& v_{n} \rightarrow v \text { in } H_{b_{\infty}}\left(\mathbb{R}^{N}\right), \\
& v_{n} \rightarrow v \text { in } L_{\text {loc }}^{2}\left(\mathbb{R}^{N}, \mathbb{C}\right), \\
& v_{n} \rightarrow v \text { a.e. on } \mathbb{R}^{N} .
\end{aligned}
$$

Using the Brezis-Lieb lemma as in [8, 25] we have

$$
1 / K_{\infty}=\|v\|_{L^{q}\left(\mathbb{R}^{N}, \mathbb{C}\right)}^{q}+\lim _{n \rightarrow \infty}\left\|w_{n}\right\|_{L^{q}\left(\mathbb{R}^{N}, \mathbb{C}\right)}^{q},
$$

where $w_{n}:=v_{n}-v$. Therefore, similarly with the proof of Theorem 1.34 of Willem [25] we have

$$
\begin{aligned}
m_{\infty} & =\lim _{n \rightarrow \infty}\left\|v_{n}\right\|_{b_{\infty}}^{2}=\|v\|_{b_{\infty}}^{2}+\lim _{n \rightarrow \infty}\left\|w_{n}\right\|_{b_{\infty}}^{2} \\
& \geq m_{\infty}\left[\left(K_{\infty}\|v\|_{L^{q}\left(\mathbb{R}^{N}, \mathbb{C}\right)}^{q}\right)^{2 / q}+\left(1-K_{\infty}\|v\|_{L^{q}\left(\mathbb{R}^{N}, \mathbb{C}\right)}^{q}\right)^{2 / q}\right] .
\end{aligned}
$$

Using (42), $v \neq 0$, we obtain $K_{\infty}\|v\|_{L^{q}\left(\mathbb{R}^{N}, \mathbb{C}\right)}^{q}=1$, and so

$$
\|v\|_{b_{\infty}}^{2}=m_{\infty}=\lim _{n \rightarrow \infty}\left\|v_{n}\right\|_{b_{\infty}}^{2} .
$$

This proof is now complete.

Consider the following Nehari manifold

$$
S^{\prime}:=\left\{u \in H_{b}\left(\mathbb{R}^{N}\right) \backslash\{0\}:\left\langle\Upsilon_{\infty}^{\prime}(u), u\right\rangle=0\right\}
$$

Lemma 5.5. Let $\Psi_{\infty}=\inf \left\{\Upsilon_{\infty}(u): u \in S^{\prime}\right\}$. Then there exists $\widehat{u} \in H_{b}\left(\mathbb{R}^{N}\right)$ such that

$$
\Psi_{\infty}=\Upsilon_{\infty}(\widehat{u})=\sup _{s \geq 0} \Upsilon_{\infty}(s \widehat{u})
$$


Proof. For all $\varphi \in H_{b}\left(\mathbb{R}^{N}\right) \backslash\{0\}$, let us define

$$
k(s)=\Upsilon_{\infty}(s \varphi)=\frac{s^{2}}{2} \int_{\mathbb{R}^{N}}\left(\left|\nabla_{A} \varphi\right|^{2}+b_{\infty}|\varphi|^{2}\right) d x-\frac{s^{q}}{q} \int_{\mathbb{R}^{N}} K_{\infty}|\varphi|^{q} d x .
$$

Since $2<q<2^{*}$, it is clear that $k(s)$ has a maximum which is a unique critical point for $k$ on $\{s: s \geq 0\}$. Moreover, taking any $\varphi$ on the set

$$
\mathcal{M}:=\left\{\varphi: \varphi \in H_{b}\left(\mathbb{R}^{N}\right), \int_{\mathbb{R}^{N}} K_{\infty}|\varphi|^{q} d x=1\right\}
$$

one immediately finds a unique element

$$
s_{\varphi}:=\left(\int_{\mathbb{R}^{N}}\left(\left|\nabla_{A} \varphi\right|^{2}+b_{\infty}|\varphi|^{2}\right) d x\right)^{\frac{1}{q-2}}
$$

such that $s_{\varphi} \varphi \in S^{\prime}$. From Lemma 5.4 we can see that the minimum of problem (41) is achieved by $v \in H_{b_{\infty}}\left(\mathbb{R}^{N}\right)$, and then using $(B)$ we can easily deduce that $v \in H_{b}\left(\mathbb{R}^{N}\right)$. So, we can conclude that there exists a unique $s_{v}=m_{\infty}^{1 /(q-2)}>0$ such that $\widehat{u}:=m_{\infty}^{1 /(q-2)} v \in S^{\prime}$ and

$$
\begin{aligned}
\Psi_{\infty} & \leq \sup _{s \geq 0} \Upsilon_{\infty}(s \widehat{u})=\Upsilon_{\infty}(\widehat{u})=\Upsilon_{\infty}\left(m_{\infty}^{1 /(q-2)} v\right) \\
& =\Upsilon_{\infty}\left(s_{v} v\right)=\sup _{s \geq 0} \Upsilon_{\infty}(s v)=\left(\frac{1}{2}-\frac{1}{q}\right) m_{\infty}^{q /(q-2)} .
\end{aligned}
$$

Now $\Psi_{\infty}$ can be easily computed in terms of $m_{\infty}$. Indeed, using $(B)$ we can obtain

$$
\begin{aligned}
\Psi_{\infty} & =\inf _{u \in S^{\prime}} \Upsilon_{\infty}(u)=\inf _{u \in S^{\prime}} \sup _{s \geq 0} \Upsilon_{\infty}(s u) \\
& \geq \inf _{u \in H_{b}\left(\mathbb{R}^{N}\right) \backslash\{0\}} \sup _{s \geq 0} \Upsilon_{\infty}(s u) \\
& =\inf _{u \in H_{b}\left(\mathbb{R}^{N}\right) \backslash\{0\}} \Upsilon_{\infty}\left(s_{u} u\right) \\
& =\left(\frac{1}{2}-\frac{1}{q}\right) m_{\infty}^{q /(q-2)}=\Upsilon_{\infty}(\widehat{u}),
\end{aligned}
$$

where

$$
s_{u}=\left(\frac{\int_{\mathbb{R}^{N}}\left(\left|\nabla_{A} u\right|^{2}+b_{\infty}|u|^{2}\right) d x}{\int_{\mathbb{R}^{N}} K_{\infty}|u|^{q} d x}\right)^{1 /(q-2)} .
$$

This proof is now complete.

Lemma 5.6. Assume $\left\{u_{n}\right\}$ is $(P S)_{c^{\prime}}$ sequence of $\Psi$ that converges weakly to $u_{0}$ in $H_{b}\left(\mathbb{R}^{N}\right)$. Then the following alternative holds: either $\left\{u_{n}\right\}$ converges strongly in $H_{b}\left(\mathbb{R}^{N}\right)$, or $c^{\prime} \geq \Psi\left(u_{0}\right)+\Psi_{\infty}$.

Proof. Since $\left\{u_{n}\right\}$ is a $(P S)_{c^{\prime}}$ sequence and $u_{n} \rightarrow u_{0}$ in $H_{b}\left(\mathbb{R}^{N}\right)$ we get

$$
\Psi\left(u_{n}\right)=c^{\prime}+o(1) \text { and }\left\langle\Psi^{\prime}\left(u_{n}\right), u_{n}\right\rangle=o(1) .
$$


We set $v_{n}=u_{n}-u_{0}$. Then $v_{n} \rightarrow 0$ in $H_{b}\left(\mathbb{R}^{N}\right)$ which yields

$$
\begin{array}{ll}
\operatorname{Re} \int_{\mathbb{R}^{N}}\left(\nabla_{A} v_{n} \overline{\nabla_{A} u_{0}}+b(x) v_{n} \overline{u_{0}}\right) d x \rightarrow 0 & \text { as } n \rightarrow \infty, \\
\operatorname{Re} \int_{\mathbb{R}^{N}} g(x) \overline{v_{n}} d x \rightarrow 0 & \text { as } n \rightarrow \infty .
\end{array}
$$

We rewrite the above relations as

$$
\begin{aligned}
& \left\|u_{n}\right\|_{b}^{2}=\left\|u_{0}\right\|_{b}^{2}+\left\|v_{n}\right\|_{b}^{2}+o(1), \\
& \Psi\left(v_{n}\right)=\Upsilon\left(v_{n}\right)+o(1) .
\end{aligned}
$$

By (44), (45) and Lemmas 5.1, 2.10, it follows that

$$
\begin{aligned}
& o(1)+c^{\prime}=\Psi\left(u_{n}\right)=\Psi\left(u_{0}\right)+\Psi\left(v_{n}\right)+o(1)=\Psi\left(u_{0}\right)+\Upsilon\left(v_{n}\right)+o(1), \\
& o(1)=\left\langle\Psi^{\prime}\left(u_{n}\right), u_{n}\right\rangle=\left\langle\Psi^{\prime}\left(u_{0}\right), u_{0}\right\rangle+\left\langle\Upsilon^{\prime}\left(v_{n}\right), v_{n}\right\rangle+o(1) \\
& =\left\langle\Upsilon^{\prime}\left(v_{n}\right), v_{n}\right\rangle+o(1) .
\end{aligned}
$$

If $v_{n} \rightarrow 0$ in $H_{b}\left(\mathbb{R}^{N}\right)$, then

$$
u_{n} \rightarrow u_{0} \text { in } H_{b}\left(\mathbb{R}^{N}\right) \text { and } \Psi\left(u_{0}\right)=\lim _{n \rightarrow \infty} \Psi\left(u_{n}\right)=c^{\prime} .
$$

If $v_{n} \not \rightarrow 0$ in $H_{b}\left(\mathbb{R}^{N}\right)$, then combining this fact that $v_{n} \rightarrow 0$ in $H_{b}\left(\mathbb{R}^{N}\right)$ we may assume that $\left\|v_{n}\right\|_{b} \rightarrow l^{\prime}$. Then (46) and Lemma 2.11 yield

$$
\begin{aligned}
c^{\prime}= & \Psi\left(u_{0}\right)+\Upsilon_{\infty}\left(v_{n}\right)+o(1), \\
\mu_{n}^{\prime}= & \left\langle\Upsilon_{\infty}^{\prime}\left(v_{n}\right), v_{n}\right\rangle=\int_{\mathbb{R}^{N}}\left(\left|\nabla_{A} v_{n}\right|^{2}+b_{\infty}\left|v_{n}\right|^{2}\right) d x \\
& -\int_{\mathbb{R}^{N}} K_{\infty}\left|v_{n}\right|^{q} d x=\alpha_{n}^{\prime}-\beta_{n}^{\prime},
\end{aligned}
$$

where

$$
\begin{aligned}
& \lim _{n \rightarrow \infty} \mu_{n}^{\prime}=0, \\
& \alpha_{n}^{\prime}=\int_{\mathbb{R}^{N}}\left(\left|\nabla_{A} v_{n}\right|^{2}+b_{\infty}\left|v_{n}\right|^{2}\right) d x \geq\left\|v_{n}\right\|_{b}^{2}, \\
& \beta_{n}^{\prime}=\int_{\mathbb{R}^{N}} K_{\infty}\left|v_{n}\right|^{q} d x \geq 0 .
\end{aligned}
$$

In light of (47), it remains to prove that $\Upsilon_{\infty}\left(v_{n}\right) \geq \Psi_{\infty}+o(1)$. For $s>0$, we obtain

$$
\left\langle\Upsilon_{\infty}^{\prime}\left(s v_{n}\right), s v_{n}\right\rangle=s^{2} \int_{\mathbb{R}^{N}}\left(\left|\nabla_{A} v_{n}\right|^{2}+b_{\infty}\left|v_{n}\right|^{2}\right) d x-s^{q} \int_{\mathbb{R}^{N}} K_{\infty}\left|v_{n}\right|^{q} d x .
$$

If we show the existence of a sequence $\left\{s_{n}\right\}$ with $s_{n} \geq 0, s_{n} \rightarrow 1$ and

$$
\left\langle\Upsilon_{\infty}^{\prime}\left(s_{n} v_{n}\right), s_{n} v_{n}\right\rangle=0
$$

then

$$
\begin{aligned}
\Upsilon_{\infty}\left(v_{n}\right) & =\Upsilon_{\infty}\left(s_{n} v_{n}\right)+\frac{1-s_{n}^{2}}{2} \alpha_{n}^{\prime}-\frac{1-s_{n}^{q}}{q} K_{\infty}\left\|v_{n}\right\|_{L^{q}\left(\mathbb{R}^{N}, \mathbb{C}\right)}^{q} \\
& =\Upsilon_{\infty}\left(s_{n} v_{n}\right)+o(1) \geq \Psi_{\infty}+o(1)
\end{aligned}
$$


and the conclusion follows. For this purpose, let $s=1+\delta^{\prime \prime}$ with $\left|\delta^{\prime \prime}\right|$ sufficiently small and employing (48) we have

$$
\begin{aligned}
\left\langle\Upsilon_{\infty}^{\prime}\left(s v_{n}\right), s v_{n}\right\rangle & =\left(1+\delta^{\prime \prime}\right)^{2} \alpha_{n}^{\prime}-\left(1+\delta^{\prime \prime}\right)^{q} \beta_{n}^{\prime} \\
& =\left(1+\delta^{\prime \prime}\right)^{2} \alpha_{n}-\left(1+\delta^{\prime \prime}\right)^{q}\left(\alpha_{n}^{\prime}-\mu_{n}^{\prime}\right) \\
& =\alpha_{n}^{\prime}\left(2 \delta^{\prime \prime}-q \delta^{\prime \prime}+o\left(\delta^{\prime \prime}\right)\right)+\left(1+\delta^{\prime \prime}\right)^{q} \mu_{n}^{\prime} \\
& =\alpha_{n}^{\prime}(2-q) \delta^{\prime \prime}+\alpha_{n}^{\prime} o\left(\delta^{\prime \prime}\right)+\left(1+\delta^{\prime \prime}\right)^{q} \mu_{n}^{\prime} .
\end{aligned}
$$

On account of $\alpha_{n}^{\prime} \rightarrow \bar{l}^{\prime} \geq l^{\prime 2}>0, \lim _{n \rightarrow \infty} \mu_{n}^{\prime}=0$ and $q>2$, then for $n$ sufficiently large, we define $\delta_{n}^{\prime \prime+}=2\left|\mu_{n}^{\prime}\right| /\left(q \alpha_{n}-2 \alpha_{n}\right)$ and $\delta_{n}^{\prime \prime-}=-2\left|\mu_{n}^{\prime}\right| /\left(q \alpha_{n}-2 \alpha_{n}\right)$, which verify the following properties:

$$
\begin{aligned}
& \delta_{n}^{\prime \prime} \searrow 0 \text { and }\left\langle\Upsilon_{\infty}^{\prime}\left(\left(1+\delta_{n}^{\prime \prime}\right) v_{n}\right),\left(1+\delta_{n}^{\prime \prime+}\right) v_{n}\right\rangle<0 \\
& \delta_{n}^{\prime \prime-} \nearrow 0 \text { and }\left\langle\Upsilon_{\infty}^{\prime}\left(\left(1+\delta_{n}^{\prime \prime}\right) v_{n}\right),\left(1+\delta_{n}^{\prime \prime-}\right) v_{n}\right\rangle>0
\end{aligned}
$$

By (49) we derive the existence of $s_{n} \in\left(1+\delta_{n}^{\prime \prime-}, 1+\delta_{n}^{\prime \prime+}\right)$ such that

$$
s_{n} \rightarrow 1 \text { and }\left\langle\Upsilon_{\infty}^{\prime}\left(s_{n} v_{n}\right), s_{n} v_{n}\right\rangle=0
$$

This completes our proof.

Let $\widehat{u} \in H_{b}\left(\mathbb{R}^{N}\right)$ satisfy (43). We can find $s_{0}^{\prime}>0$ such that

$$
\begin{aligned}
& \Upsilon(s \widehat{u})<0 \text { if } s \geq s_{0}^{\prime}, \\
& \Psi(s \widehat{u})<0 \text { if } s \geq s_{0}^{\prime} \text { and }\|g\|_{b,-1} \leq 1 .
\end{aligned}
$$

We shall define

$$
\begin{aligned}
& \mathcal{P}^{\prime}=\left\{\gamma \in C\left([0,1], H_{b}\left(\mathbb{R}^{N}\right)\right) \mid \gamma(0)=0, \gamma(1)=s_{0}^{\prime} \widehat{u}\right\} \\
& c_{1}^{\prime}=\inf _{\gamma \in \mathcal{P}^{\prime}} \sup _{u \in \gamma} \Psi(u) .
\end{aligned}
$$

Lemma 5.7. There are $R_{1}^{\prime}>0, C^{\prime}=C\left(R_{1}^{\prime}\right)>0$ and $\delta_{R_{1}^{\prime}}>0$ such that for all $g$ with $\|g\|_{b,-1}<C^{\prime}$ we have $\left.\Psi\right|_{\partial B_{R_{1}^{\prime}}} \geq \delta_{R_{1}^{\prime}}$ and $c_{1}^{\prime}<c_{0}^{\prime}+\Psi_{\infty}$, where $c_{1}^{\prime}$ is given by $(51)$ and $c_{0}^{\prime}=\inf _{\bar{B}_{R_{1}^{\prime}}} \Psi(u)$.

Proof. Using conditions $(M)$ and the definition of $\Upsilon$ we may assume that $\Upsilon(s \widehat{u})<$ $\Upsilon_{\infty}(s \widehat{u})$ for all $s>0$. A direct computation yields that there is $\widehat{s} \in\left(0, s_{0}^{\prime}\right)$ such that

$$
\sup _{s \geq 0} \Psi(s \widehat{u})=\Psi(\widehat{s} \widehat{u})<\Upsilon_{\infty}(\widehat{s} \widehat{u}) \leq \sup _{s \geq 0} \Upsilon_{\infty}(s \widehat{u})=\Psi_{\infty}
$$

Then, there is a constant $\varepsilon_{1}^{\prime} \in(0,1)$ such that

$$
\sup _{s \geq 0} \Upsilon(s \widehat{u})<\Psi_{\infty}-\varepsilon_{1}^{\prime} .
$$

For this $\varepsilon_{1}^{\prime}$, we obtain the existence of $R_{1}^{\prime}>0$ and $C_{1}^{\prime}=C_{1}^{\prime}\left(\varepsilon_{1}^{\prime}\right)=C_{1}^{\prime}\left(R_{1}^{\prime}\right)>0$ such that for all $g \not \equiv 0$ and $\|g\|_{b,-1}<C_{1}^{\prime}$ the conclusion of Lemma 5.2 holds true. 
Furthermore, by virtue of its proof, there is $\delta_{R_{1}^{\prime}}>0$ such that $\left.\Psi\right|_{\partial B_{R_{1}^{\prime}}} \geq \delta_{R_{1}^{\prime}}$, provided that $\|g\|_{b,-1}<C_{1}^{\prime}$. Choosing $C_{2}^{\prime}=\min \left\{C_{1}^{\prime}, \varepsilon_{1}^{\prime} \sqrt{\varepsilon_{1}^{\prime}}\right\}$ we find

$$
c_{0}^{\prime}=\inf _{u \in \bar{B}_{R_{1}^{\prime}}} \Psi(u) \geq-\frac{1}{2 \varepsilon_{1}^{\prime 2}}\|g\|_{b,-1}>-\frac{\varepsilon_{1}^{\prime}}{2} \text { for all } g \text { with }\|g\|_{b,-1} \leq C_{2}^{\prime} .
$$

If $\|g\|_{b,-1}<\varepsilon_{1}^{\prime} /\left(2 s_{0}^{\prime}\|\widehat{u}\|_{b}\right)$, then for $\gamma_{0}=\left\{s s_{0}^{\prime} \widehat{u}: 0 \leq s \leq 1\right\}$ we have

$$
|\Psi(u)-\Upsilon(u)|=\left|\int_{\mathbb{R}^{N}} g(x) u d x\right| \leq s_{0}^{\prime} \int_{\mathbb{R}^{N}} g(x) \widehat{u} d x \leq s_{0}^{\prime}\|\widehat{u}\|_{b}\|g\|_{b,-1}<\frac{\varepsilon_{1}^{\prime}}{2} .
$$

So, if $\|g\|_{b,-1}<C^{\prime}=\min \left\{C_{2}^{\prime}, \varepsilon_{1}^{\prime} /\left(2 s_{0}^{\prime}\|\widehat{u}\|_{b}\right)\right\}$ then for all $g \not \equiv 0$ with $\|g\|_{b,-1}<C^{\prime}$ we obtain

$$
\Psi(u)<\Upsilon(u)+\frac{\varepsilon_{1}^{\prime}}{2} \text { for } u \in \gamma_{0},
$$

and from (50), (52), (53) it follows that

$$
\begin{aligned}
c_{1}^{\prime} & =\inf _{\gamma \in \mathcal{P}^{\prime}} \sup _{u \in \gamma} \Psi(u) \leq \sup _{u \in \gamma_{0}} \Psi(u) \leq \sup _{u \in \gamma_{0}} \Upsilon(u)+\frac{\varepsilon_{1}^{\prime}}{2} \\
& \leq \sup _{s \geq 0} \Upsilon(s u)<\Psi_{\infty}-\frac{\varepsilon_{1}^{\prime}}{2} \leq \Psi_{\infty}+c_{0} .
\end{aligned}
$$

This completes our proof.

\subsection{Proof of Theorem 2.6 completed}

Consider $R_{1}^{\prime}>0, C^{\prime}=C^{\prime}\left(R_{1}^{\prime}\right)>0$ and $\delta_{R_{1}^{\prime}}>0$ given by Lemma 5.7, in view of its proof, we get that for all $g \not \equiv 0$ with $\|g\|_{b,-1}<C^{\prime}$ the conclusion of Lemma 5.2 also holds. So, we get the existence of a solution $u_{0}^{\prime} \in H_{b}\left(\mathbb{R}^{N}\right)$ of problem (3) such that $\Psi\left(u_{0}^{\prime}\right)=c_{0}^{\prime}$.

Furthermore, it follows from the Mountain Pass Theorem without the PalaisSmale condition $\left[9\right.$, Theorem 2.2] that there exists a sequence $\left\{u_{n}\right\}$ such that

$$
\Psi\left(u_{n}\right)=c_{1}^{\prime}+o(1) \text { and } \Psi^{\prime}\left(u_{n}\right) \rightarrow 0 \text { in } H_{b}^{-1}\left(\mathbb{R}^{N}\right) .
$$

This yields

$$
\begin{aligned}
& c_{1}^{\prime}+o(1)+\frac{1}{q}\left\|\Psi^{\prime}\left(u_{n}\right)\right\|_{b,-1}\left\|u_{n}\right\|_{b} \\
\geq & \Psi\left(u_{n}\right)-\frac{1}{q}\left\langle\Psi^{\prime}\left(u_{n}\right), u_{n}\right\rangle \\
\geq & \left(\frac{1}{2}-\frac{1}{q}\right)\left\|u_{n}\right\|_{b}^{2}-\left(1-\frac{1}{q}\right)\|g\|_{b,-1}\left\|u_{n}\right\|_{b} .
\end{aligned}
$$

Therefore $\left\{u_{n}\right\}$ is bounded sequence in $H_{b}\left(\mathbb{R}^{N}\right)$ and, passing to subsequence, we may suppose that $u_{n} \rightarrow u_{1}^{\prime}$ in $H_{b}\left(\mathbb{R}^{N}\right)$ for some $u_{1}^{\prime} \in H_{b}\left(\mathbb{R}^{N}\right)$. Thus, using Lemma $5.1, u_{1}^{\prime}$ is a weak solution of problem (3). 
Finally, we prove that $J\left(u_{0}^{\prime}\right) \neq J\left(u_{1}^{\prime}\right)$. Indeed, from Lemma 5.6, either $u_{n} \rightarrow u_{1}^{\prime}$ in $H_{b}\left(\mathbb{R}^{N}\right)$ which gives

$$
\Psi\left(u_{1}^{\prime}\right)=\lim _{n \rightarrow \infty} \Psi\left(u_{n}\right)=c_{1}^{\prime}>0>c_{0}^{\prime}=\Psi\left(u_{0}^{\prime}\right)
$$

and the conclusion follows, or

$$
c_{1}^{\prime}=\lim _{n \rightarrow \infty} \Psi\left(u_{n}\right) \geq \Psi\left(u_{1}^{\prime}\right)+\Psi_{\infty} .
$$

If we assume that $\Psi\left(u_{1}^{\prime}\right)=\Psi\left(u_{0}^{\prime}\right)=c_{0}^{\prime}$, then $c_{1}^{\prime} \geq c_{0}^{\prime}+\Psi_{\infty}$ which contradicts Lemma 5.7 .

This proof of Theorem 2.6 is now complete.

\section{Acknowledgment}

The authors would like to thank the anonymous referee for the careful reading of the manuscript and for numerous comments which improved the initial version of this paper. This paper has been completed while Youpei Zhang was visiting University of Craiova (Romania) with the financial support of the China Scholarship Council (No. 201906370079). Youpei Zhang would like to thank the China Scholarship Council and the Embassy of the People's Republic of China in Romania.

Open Access. This article is licensed under a Creative Commons Attribution 4.0 International License, which permits use, sharing, adaptation, distribution and reproduction in any medium or format, as long as you give appropriate credit to the original author(s) and the source, provide a link to the Creative Commons licence, and indicate if changes were made. The images or other third party material in this article are included in the article's Creative Commons licence, unless indicated otherwise in a credit line to the material. If material is not included in the article's Creative Commons licence and your intended use is not permitted by statutory regulation or exceeds the permitted use, you will need to obtain permission directly from the copyright holder. To view a copy of this licence, visit http://creativecommons.org/licenses/by/4.0/.

Publisher's Note. Springer Nature remains neutral with regard to jurisdictional claims in published maps and institutional affiliations.

\section{References}

[1] C.O. Alves, G.M. Figueiredo and M.F. Furtado, Multiple solutions for a nonlinear Schrödinger equation with magnetic fields, Comm. in Partial Differential Equations 36 (2011), 1565-1586.

[2] C.O. Alves and G.M. Figueiredo, Multiple solutions for a semilinear elliptic equation with critical growth and magnetic field, Milan J. Math. 82 (2014), 389-405.

[3] A. Ambrosetti and P. Rabinowitz, Dual variational methods in critical point theory and applications, J. Funct. Anal. 14 (1973), 349-381.

[4] G. Arioli and A. Szulkin, A semilinear Schrödinger equation in the presence of a magnetic field, Arch. Ration. Mech. Anal. 170 (2003), 277-295. 
[5] A. Bahri, Topological results on a certain class of functionals and applications, J. Funct. Anal. 41 (1981), 397-427.

[6] A. Bahri and H. Berestycki, A perturbation method in critical point theory and applications, Trans. Amer. Math. Soc. 267 (1981), 1-32.

[7] H. Brezis, Analyse Fonctionnelle: Théorie et Applications, Masson, Paris, 1983.

[8] H. Brezis and E.H. Lieb, A relation between pointwise convergence of functions and convergence of functionals, Proc. Amer. Math. Soc. 88 (1983), 486-490.

[9] H. Brezis and L. Nirenberg, Positive solutions of nonlinear elliptic equations involving critical Sobolev exponent, Comm. Pure Appl. Math. 36 (1983), 437-477.

[10] F. Cîrstea and V.D. Rădulescu, Multiple solutions of degenerate perturbed elliptic problems involving a subcritical Sobolev exponent, Topol. Methods Nonlinear Anal. 15 (2000), 283-300.

[11] M. Clapp and A. Szulkin, Multiple solutions to a nonlinear Schrödinger equation with Aharonov-Bohm magnetic potential, NoDEA Nonlinear Differential Equations Appl. 17 (2010), 229-248.

[12] M.J. Esteban and P.L. Lions, Stationary solutions of nonlinear Schrödinger equations with an external magnetic field, in: Partial Differential Equations and the Calculus of Variations, vol. 1 (F. Colombini, A. Marino, L. Modica and S. Spagnolo, eds.), Birkhäuser, 1989, pp. 401-449.

[13] C. Ji and V.D. Rădulescu, Multi-bump solutions for the nonlinear magnetic Schrödinger equation with exponential critical growth in $\mathbb{R}^{2}$, Manuscripta Math. (2020) https://doi.org/10.1007/ s00229-020-01195-1.

[14] C. Ji and V.D. Rădulescu, Multiplicity and concentration of solutions to the nonlinear magnetic Schrödinger equation, Calc. Var. Partial Differential Equations 59 (2020), no. 4, paper No. 115, 28 pp.

[15] A. Laptev and S. Maad Sasane, Perturbations of embedded eigenvalues for a magnetic Schrödinger operator on a cylinder, J. Math. Phys. 58 (2017), no. 1, 012105, 17 pp.

[16] A. Laptev, M. Ruzhansky and N. Yessirkegenov, Hardy inequalities for Landau Hamiltonian and for Baouendi-Grushin operator with Aharonov-Bohm type magnetic field. Part I, Math. Scand. 125 (2019), no. 2, 239-269.

[17] H. Leinfelder, Gauge invariance of Schrödinger operators and related spectral properties, J. Operator Theory 9 (1983), 163-179.

[18] E.H. Lieb and M. Loss, Analysis, Graduate Studies in Mathematics, vol. 14, American Mathematical Society, Providence, RI, 1997.

[19] N.S. Papageorgiou, V.D. Rădulescu and D.D. Repovš, Nonlinear Analysis-Theory and Methods, Springer Monographs in Mathematics, Springer, Cham, 2019.

[20] P. Pucci and V.D. Rădulescu, The impact of the mountain pass theory in nonlinear analysis: a mathematical survey, Boll. Unione Mat. Ital. 9 (2010), no. 3, 543-582.

[21] P.H. Rabinowitz, Minimax Methods in Critical Point Theory with Applications to Differential Equations, CBMS Reg. Conf. Ser. Math., vol. 65, American Mathematical Society, Providence, RI, 1986.

[22] V.D. Rădulescu and D. Smets, Critical singular problems on infinite cones, Nonlinear Anal. 54 (2003), 1153-1164. 
[23] M. Struwe, Infinitely many critical points for functionals which are not even and applications to superlinear boundary value problems, Manuscripta Math. 32 (1980), 335-364.

[24] M. Struwe, Variational Methods: Applications to Nonlinear Partial Differential Equations and Hamiltonian Systems, Ergebnisse der Mathematik und ihrer Grenzgebiete, A Series of Modern Surveys in Mathematics, vol. 34, Springer-Verlag, Berlin, 2008.

[25] M. Willem, Minimax Theorems, Progress in Nonlinear Differential Equations and Their Applications, vol. 24, Birkhäuser Boston, Inc., Boston, MA, 1996.

Youpei Zhang

School of Mathematics and Statistics, Central South University

Changsha, Hunan 410083, P.R. China

and

Department of Mathematics, University of Craiova

Street A.I. Cuza No. 13, 200585 Craiova, Romania

e-mail: zhangypzn@163.com

Xianhua Tang

School of Mathematics and Statistics, Central South University

Changsha, Hunan 410083, P.R. China

e-mail: tangxh@mail.csu.edu.cn

Vicenţiu D. Rădulescu*

Department of Mathematics, University of Craiova

Street A.I. Cuza No. 13, 200585 Craiova, Romania

and

Faculty of Applied Mathematics, AGH University of Science and Technology

al. Mickiewicza 30, 30-059 Krakow, Poland

e-mail: radulescu@inf.ucv.ro

Received: May 8, 2020.

Revised: September 9, 2020.

Accepted: September 24, 2020. 